\title{
Assessing learning in small sized physics courses
}

\author{
Emanuela Ene* \\ Department of Physics \& Astronomy, Texas A\&M University, College Station, Texas 77843-4242, USA \\ Bruce J. Ackerson ${ }^{\dagger}$ \\ Department of Physics, Oklahoma State University, Stillwater, Oklahoma 74078-3072, USA
}

(Received 15 August 2017; published 18 January 2018)

\begin{abstract}
We describe the construction, validation, and testing of a concept inventory for an Introduction to Physics of Semiconductors course offered by the department of physics to undergraduate engineering students. By design, this inventory addresses both content knowledge and the ability to interpret content via different cognitive processes outlined in Bloom's revised taxonomy. The primary challenge comes from the low number of test takers. We describe the Rasch modeling analysis for this concept inventory, and the results of the calibration on a small sample size, with the intention of providing a useful blueprint to other instructors. Our study involved 101 students from Oklahoma State University and fourteen faculty teaching or doing research in the field of semiconductors at seven universities. The items were written in four-option multiple-choice format. It was possible to calibrate a 30 -item unidimensional scale precisely enough to characterize the student population enrolled each semester and, therefore, to allow the tailoring of the learning activities of each class. We show that this scale can be employed as an item bank from which instructors could extract short testlets and where we can add new items fitting the existing calibration.
\end{abstract}

DOI: 10.1103/PhysRevPhysEducRes.14.010102

\section{INTRODUCTION}

The concept examination, described here, grew from the desire to assess the effectiveness of a new pedagogy for a junior level course PHYS3313 at Oklahoma State University (OSU). The course was initially a standard, one-semester, introductory modern physics course. Since the enrollment evolved to include mainly students in electrical and computer engineering, the departments of physics and electrical engineering collaborated to change the emphasis to an introduction to solid-state devices with a nontraditional pedagogy described by Cheville and Bunting [1,2]. Currently, as different instructors are assigned to teach this class, the pedagogy shifts between traditional and nontraditional forms.

There were few assessment instruments for specialty courses in 2009 and none suitable for our PHYS3313 course. Because of the wide use of concept inventories for assessment ${ }^{1}$

\footnotetext{
*ene@physics.tamu.edu

bruce.ackerson@okstate.edu

${ }^{1}$ Among the popular concept inventories, we mention the Force Concept Inventory (FCI) that discriminates Newtonian from nonNewtonian thinkers. There is a plethora of concept inventories for a variety of disciplines. The effort to develop such assessment instruments has helped identify what are the most important concepts and cognitive skills targeted by similar courses taught by different instructors.
}

Published by the American Physical Society under the terms of the Creative Commons Attribution 3.0 License. Further distribution of this work must maintain attribution to the author(s) and the published article's title, journal citation, and DOI.
[3-7], building and calibrating a concept inventory arose as the natural way to obtain a reference knowledge scale for comparing different pedagogies. Concept inventories aim at measuring knowledge and knowledge interpretation. Citing Reif [8,9], conceptual knowledge is the kind of knowledge that may be transferred between situations and can be used for inference; it can be achieved through reflective learning. For successfully solving the collection of problems in a concept inventory, one needs not only conceptual knowledge but also factual, procedural, and metacognitive ${ }^{2}$ knowledge as well as the skills required to operate effectively with knowledge [10]. We interpreted problem solving as a succession of operations, some as simple as recalling, some as complex as analyzing cause-and-effect statements. For the purpose of problem writing, we developed operational definitions of the cognitive levels in Bloom's revised taxonomy [11,12].

During the early phase of field testing open-response problems, we realized that due to low enrollment in the physics of semiconductors class at OSU we would collect no more than 15 responses to the concept inventory per semester. Classical test calibration on large size samples of persons was not a possible alternative in our case and therefore we turned our attention to the Item Response Theory (IRT). We adopted the Rasch model [13] as the method best suited for calibrating a reference knowledge scale on a small size sample

\footnotetext{
${ }^{2}$ Metacognition, which is confidence in one's own knowledge, assures consistency in applying available knowledge. A typical novice student lacks that consistency that facilitates debugging and helps regenerate knowledge that is partially forgotten. It is the metacognition that novice students are often missing.
} 
of persons. The Rasch model is sometimes called the oneparameter IRT logistic model [14].

This paper is structured as follows. Section II discusses the theoretical method that we adopted for item calibration and learning-gain measurement. Section III describes the stages of the test development and exemplifies how Bloom's taxonomy was put to work. Section IV presents the data analysis, instrument calibration, and learning-gain estimation. We show that the Rasch calibration method that we adopted was better suited than other IRT models. Section V summarizes the merits of our study and how others could benefit from it. The interested reader can find more information and results in the Supplemental Material [15].

\section{THEORETICAL MODEL FOR INVARIANT ITEM-SCALE CALIBRATION AND LEARNING-GAIN MEASUREMENT}

\section{A. Person free item calibration on a small sample}

The probabilistic model for knowledge tests, first introduced by Georg Rasch, separates the calibration of the items in the test from the population distribution. "...we have no reason for considering a normal distribution of test scores as evidence for equality of units" says Rasch [16] when comparing the classical test theory to his model. We expand on this citation: suppose we have a collection of rulers that are not very good, but we want a standard scale to measure length. First, we mark the length of each ruler on a piece of paper, keeping the opposite end of the ruler up against the wall and the paper fixed. After many markings, the density of marks approximates a Gaussian distribution; the density of marks is very dense for the average ruler length and less dense for shorter or longer rulers. We take the average length as the standard length. Alternatively, we find a ruler that has not been damaged or bent to serve as our model length scale. Then we measure all the rulers against this scale. We keep all the rulers that match the ideal and throw away the rest. These are two very different ways for determining a length scale. Classical statistical methods are captured in the first method, while the second method characterizes the Rasch model. Data determine the model parameters in the first case, while a model of measurement requires data to fit the selected model in the second case. The Rasch procedure determines an operational condition that data must satisfy in order to be measured objectively. In practice, the log of odds data must be a linear function of items' and persons' parameters to determine objectivity. If some data do not satisfy linearity, they cannot be measured by this technique (a ruler in different units, for example). Like measurements in physical science, these data are operationally defined.

As Wright [16] puts it: "When a man says he is in the 90th percentile in math ability, we need to know in what group and what test before we can make any sense of his statement. But when he says he is 5' 11' tall, we don't ask to see the yardstick". A test with the items calibrated to be invariant across various samples of the population has the specific objectivity of a yardstick, as opposed to a calibration assuming a normal distribution of the scores.

Rasch modeling increasingly finds use in concept inventory analyses [17,18] and some authors [19] plead for its use as opposed to classical test theory. One of the benefits of the Rasch modeling is that it does not require large sample size. A foundational book in Rasch modeling, "Best Test Design" [20], is based on the analysis of a sample of 35 children and 18 dichotomous items. According to Linacre, a world leader of Rasch measurement research, the minimum sample size for calibration of an objective scale is related to the desired range of stability of the item parameters [21]. The recommended minimum sample size range for a $95 \%$ confidence is 30 for $\pm 1 \operatorname{logit}^{3}$ stability and 100 for $\pm 1 / 2$ logit. "As a rule of thumb, at least 8 correct and 8 incorrect responses are needed for reasonable confidence that an item calibration is within 1 logit of a stable value" [21]. One recent study employed Rasch modeling to analyze a 24-item test, the Relativity Concept Inventory [22]. The Relativity Concept Inventory was calibrated on a small sample size: 70 persons at pretest, 63 at post-test, with 53 matched repeated measures.

\section{B. Item and person invariant parametrizations}

The Rasch model assumes that answering an item in a knowledge test is a stochastic process similar to hurdling [23] and that the probability to answer an item depends on two unknown parameters: the ability of each person and the difficulty of each test item. Neither of these parameters can be determined by direct measurement. In this paper we adopt the symbols $\theta_{\nu}$ for person parameter (ability) and $\beta_{i}$ for item parameter (difficulty). Assuming statistical independence of the items and of the answers, the probability of a person $\nu$ to answer a dichotomous item $i$ is given by

$$
P[i, \nu]=\frac{e^{x_{i \nu}\left(\theta_{\nu}-\beta_{i}\right)}}{1+e^{\left(\theta_{\nu}-\beta_{i}\right)}},
$$

where $x_{i \nu}$ takes the value 0 if the answer is incorrect, and 1 if correct [24]. So, when $x_{i \nu}=1$ we have the probability that the person $\nu$ answers question $i$ correctly. Fisher [25] demonstrates that such a model can be calibrated to produce an objective scale, which orders the difficulty of the items independent of the test takers. Likewise, because of the symmetry of the probability function, the population of test takers becomes ordered according to ability. To place the item parameters on an interval scale requires only the "sufficient statistic" of the raw test score from each person (a sum over item scores for each person) [26].

\footnotetext{
${ }^{3} 1$-logit is the unit of measure for the parameters estimated via logistic models. 1-logit signifies that the natural logarithm of the odds of an event equals one. This is equivalent to a probability of $e /(1+e) \approx 73 \%$ to observe the event.
} 
The theoretical condition (1) can be rewritten

$$
\ln \left(\frac{P[i, \nu]}{1-P[i, \nu]}\right)=\theta_{\nu}-\beta_{i}
$$

The ratio $P[i, \nu] /(1-P[i, \nu])$ is the odds that person $\nu$ answers item $i$ correctly rather than incorrectly. The log of odds is usually called logit. Equation (2) clarifies why the dimensionless parameters $\theta_{\nu}$ and $\beta_{i}$ are measured in logit units.

Given the parameter values $\vec{\theta}=\left\{\theta_{\nu}\right\}$ and $\vec{\beta}=\left\{\beta_{i}\right\}$, and given that the assumption of independence is met [27], the probability of a collective outcome $\vec{x}=\left\{x_{i \nu}\right\}$ for $N$ persons and $K$ items can be calculated as the product

$$
P(\vec{x} \mid \vec{\beta}, \vec{\theta})=\prod_{\nu=1}^{N} \prod_{i=1}^{K} \frac{\exp \left[b_{i \nu} x_{i \nu}\left(\theta_{\nu}-\beta_{i}\right)\right]}{\left[1+\exp \left(\theta_{\nu}-\beta_{i}\right)\right]^{b_{i \nu}}},
$$

where the parameter $b_{i \nu}$ is 1 if person $\nu$ submitted an answer to item, $i$, and 0 otherwise. However, we have recorded the observations $\left\{x_{i \nu}\right\}$ and wish to estimate the parameter sets $\left\{\beta_{i}\right\}$ and $\left\{\theta_{\nu}\right\}$. This can be accomplished by maximizing the likelihood $L(\vec{\beta}, \vec{\theta} \mid \vec{x})$, or the logarithm of the likelihood, with respect to the parameters $\left\{\theta_{\nu}\right\}$ and $\left\{\beta_{i}\right\}$. The astute reader will recognize the correspondence with maximizing the entropy in statistical mechanics.

Three distinct theoretical approaches to the Rasch item parametrization have been developed: joint (JML), conditional (CML), and marginal (MML) likelihood maximization; separability is the key issue differentiating these methods. The theoretical advantage of the CML calibration, which we adopted for our inventory, is the person free item parametrization that satisfies Rasch's idea of specific objectivity [28-30]. The item parameter estimates obtained by using different subgroups (i.e., gender or ethnic groups of the population, groups at different score levels) should be equal, for an invariant calibration [31,32].

Out of the many methods to estimate the person parameters, we adopted a computationally simple marginal likelihood [33]. The invariant set of item parameters $\hat{\beta}_{i}$ obtained previously and the persons' raw scores $r_{\nu}$ are employed to find the persons' ability $\theta_{\nu}$ values. The parameter $\theta_{\nu}$ is the root of the following equation:

$$
r_{\nu}-\sum_{i=1}^{k} \frac{\exp \left(\theta_{\nu}-\hat{\beta}_{i}\right)}{1+\exp \left(\theta_{\nu}-\hat{\beta}_{i}\right)}=0 .
$$

The standard error of estimation (SEM) for the item and person parameters is minimal in the midrange where the information has a maximum $[34,35]$.

We illustrate how the probabilistic Rasch model ranks the items' difficulties and the persons' abilities on a common logit scale in Figs. 1 and 2. The common itemperson scale has the advantage of estimating the chance that

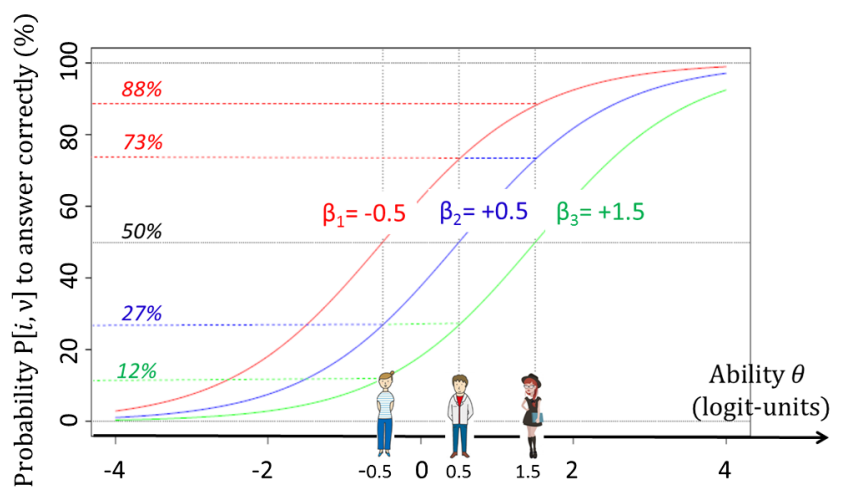

FIG. 1. Probabilistic model of the interaction person question. The probability of giving a correct answer to an item $i$ depends nonlinearly on the difference between the item's difficulty and person's ability $\left(\beta_{i}-\theta_{\nu}\right)$. The parameter $\beta_{i}$ has positive large positive values for difficult items and low negative values for easy items. The $s$-shaped logistic curves in this diagram are giving the probabilities of answering correctly for persons of different abilities $\theta_{\nu}$. The person ability is equal to the difficulty of the item with a $50 \%$ probability to be solved correctly by that person. The three persons in this cartoon have the abilities $\theta_{1}=-0.5$, $\theta_{2}=0.5$, and $\theta_{3}=1.5$. All the persons agree that the item of $\beta_{3}=1.5$ is the most difficult because it has the lowest probability to be solved correctly. If taking these persons in the increasing order of their ability, their probabilities to solve the item of 1.5logit difficulty are $12 \%, 27 \%$, and $50 \%$, respectively. Similarly, all these persons agree that the item of $\beta_{1}=-0.5$ is the easiest because they all have the highest probability to solve it correctly; these probabilities are $50 \%, 73 \%$, and $88 \%$. Only the items that are ranked in the same order, from the easiest to the most difficult, by all the persons in the sample, are retained in the Rasch invariant scale.

a person gets the correct answer only from knowing the person's location with respect to that question. On the common item-person axis, zero ability does not imply the absence of a person's ability but only a fixed point on the axis, which is usually chosen at the median of the estimated item difficulties, $\tilde{\beta}=0$. When the person's parameter $\theta_{\nu}$ equals item's difficulty $\beta_{i}$, in other words when the person and the item have the same location on the logit scale, the probability of a correct answer is $50 \%$. If the person's location is at the left of the item, the probability to answer correctly that item is less than $50 \%$; vice versa, the person located at the right of the item has more than a $50 \%$ chance to answer correctly.

From the data at hand, we can approximate the average probability of obtaining a correct answer to item $i$ through the proportion $p_{i}$ of correct answers given by all the persons in field testing. We call $p_{i}$ the item popularity. ${ }^{4}$ Item popularity characterizes globally the sample in field

\footnotetext{
${ }^{4}$ Item popularity represents the percentage of correct answers obtained by dividing the count of correct to the count of submission for this item.
} 


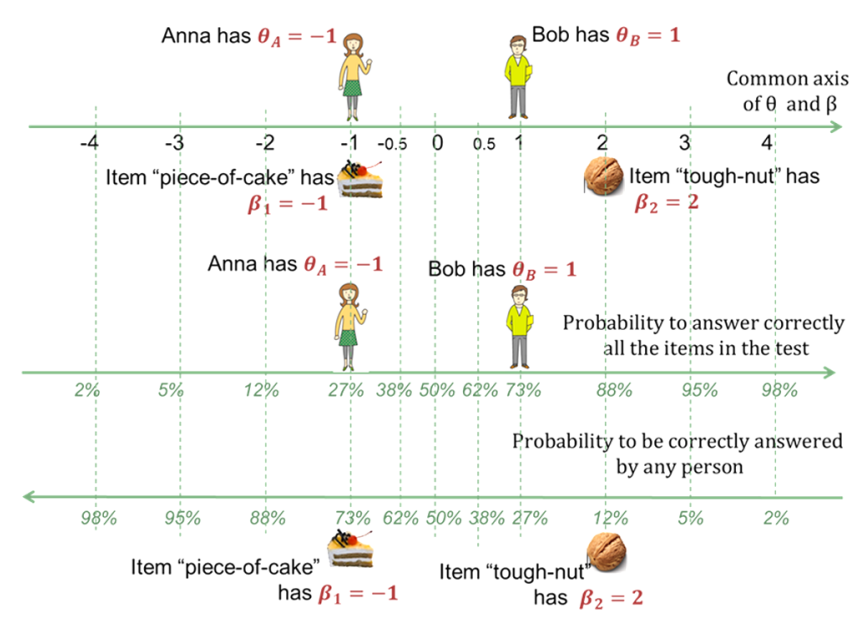

FIG. 2. The logit-unit axis simplifies the predictions with Rasch calibrated tests. Two persons, Anna and Bob, and two items, piece of cake and tough nut, are shown. From each persons' location on the common logit axis at the top, we know that Anna has a lower probability than Bob to solve correctly any item. We know that Anna has a 50\% chance to solve correctly the item piece of cake. We need Eq. (1) for calculating that Bob has an $88 \%$ chance to get piece of cake correctly, but only a $27 \%$ to solve tough nut. Anna's chance with tough nut is of $4.74 \%$. Alternately, we could refer to two separate probability axes going in opposite directions, one for persons and one for items. Instead of reading "Bob's ability is of 1 logit," one may prefer to read on the middle axis that "Bob has a $73 \%$ chance to solve correctly all the items in the test". On the lower axis going from right to left, the probabilities to correctly solve the items are shown as percentages. An average person receiving the basic training in the field measured by the test has a probability of $73 \%$ to solve the item piece of cake but only of $12 \%$ to solve the item tough nut.

testing; therefore, in Eq. (2) we substitute the unknown individual abilities by the median ability $\tilde{\theta}$ of the sample, and the item parameter by its fitted value $\hat{\beta}_{i}$. The ratio $p_{i} /\left(1-p_{i}\right)$ is the odds to answer correctly rather than incorrectly to the item $i$. Therefore, if the items fit the Rasch model, their parameters must be in a linear relationship with the observed logits of the items

$$
\ln \left(\frac{p_{i}}{1-p_{i}}\right)=\tilde{\theta}-\hat{\beta}
$$

Equation (5) predicts that the observed logits of the items fitting the model would fall on a line of slope -1 . The reader should be aware that the computational capabilities available nowadays can accurately work through exact equations like Eq. (3) and not sample dependent approximations like Eq. (5).

The set of calibrated items measures persons objectively if these persons have abilities in the specific field of the test and if the persons' locations are inside the region populated by items. If the persons have no training in the field of knowledge that is tested or if they have a significant level of expertise, they are out of target range and cannot be discriminated by the existing set of Rasch calibrated items. The person's estimated ability should not depend on the specific items chosen from a large calibrated item pool. As all the items in a Rasch calibrated test have equal discrimination, it does not matter what items are selected for estimating the ability of the respondents. Items may then be selected to align better with the person's ability in that sample. Any subset of calibrated items can be chosen for building testlets centered on the hypothesized ability of the sample of persons to be measured. These testlets should contain just enough items that would render the error of the ability estimation at a value of practical interest [36].

The Rasch calibrated test is scalable: a test bank may be developed and expanded with items of known content and difficulty [37]. New items can be developed to separate more finely the abilities of a target group. Or, in computer adaptive testing [38], a fine estimation may be done for each individual person by successively narrowing the pool of items. Writing items dedicated to different levels in Bloom's taxonomy leads to subsets of calibrated Rasch scales. The real issue becomes starting the process individually and collaboratively making an item pool to which others may add.

\section{Learning-gain estimation}

Ultimately, our interest is to characterize the population enrolled each semester (formative diagnostic), which would allow custom tailoring the teaching activities, and to find a measure of the learning gain at the end of the instructional period. The individual learning gain for a Rasch instrument is simply estimated by the change of the person parameter between pre- and post-test:

$$
\Delta \hat{\theta}=\hat{\theta}_{\mathrm{post}}-\hat{\theta}_{\mathrm{pre}}
$$

This Rasch metric for the learning gain is individual, specific, and objective because the ability gain compares successive locations of a person on a fixed reference scale. One easily notices that the $\Delta \hat{\theta}$ individual ability gain must be significantly greater than the error of estimation if we claim individual learning as a result of the instruction in that course [39-41].

During the past two decades, many publications have reported the average normalized score gain $\langle g\rangle$ as a group metric for learning gain [42]:

$\langle g\rangle=(\%\langle$ scorepost $\rangle-\%\langle$ scorepre $\rangle) /(1-\%\langle$ scorepre $\rangle)$.

On the one hand, a metric referring to a change of the score for a set of problems does not capture information of what questions were answered correctly at pre- or at postinstruction, and what questions were not answered at 
all. On the other hand, the $\langle g\rangle$ metric cannot be applied to the Rasch estimated abilities (instead of the raw scores) because the theoretical upper limit of a person's ability is infinity. In addition, some studies claim that the $\langle g\rangle$ metric is strongly dependent on the scores at preinstruction $[43,44]$. Instead of the $\langle g\rangle$ metric, we suggest to report an average ability gain $\langle\Delta \hat{\theta}\rangle$ separately for categories of persons situated at preinstruction at the same location on the calibrated item-scale. We will exemplify the group ability gain $\langle\Delta \hat{\theta}\rangle$ suggested metric in this paper.

\section{TEST DEVELOPMENT AND METHODOLOGY}

\section{A. Selection of content area}

The physics of semiconductors concept inventory (PSCI) instrument was built for measuring the ability of a person to operate with principles, laws, and idealized theoretical models related to the physics of semiconductors. Twelve experts from seven universities each provided a list of the most important concepts for the physics of semiconductors. A survey of syllabi and textbooks [45-53] currently used for introductory courses of the physics of semiconductors at U.S. universities provided the most often cited concepts in the problems proposed at the end of the chapters. We built a frequency chart of the entries from experts and books and retained the most frequent ten concept categories for item writing. ${ }^{5}$

\section{B. Item writing and revision}

Item stems ${ }^{6}$ were written both in question format and as open-ended sentences. One-third of the stems contain visual stimuli: photographs, diagrams, and graphs. One-quarter of the items have options ${ }^{7}$ in graphical format. Items were labeled with a unique identifier, giving the concept category and the position in the sequence. For example, item 8.2 pertains to the concept 8 -Statistics ${ }^{8}$ and is the second in the sequence of the items written for this concept.

For item writing, we employed the operational definitions that we created for the cognitive processes in Bloom's taxonomy. The four-option dichotomous items were written for measuring a single component of the knowledge dimension, conceptual knowledge, and four components of the cognitive dimension: understanding, application, analysis, and evaluation. Each item was assigned to the highest level of the cognitive process necessary to reach the correct answer. Most of the distracters were chosen from

\footnotetext{
${ }^{5}$ The reader can find the concept frequency chart and the list of contributors in the Supplemental Material [15].

${ }^{6}$ The stem of a multiple-choice item is the text of the problem to be solved.

${ }^{7}$ The options of a multiple choice item are the alternate answers listed. The incorrect answer options are called distracters.

${ }^{8}$ The numerical identifiers of the items and concept categories appear in bold. The names of the specific, well-defined terms in our framework are capitalized.
}

answers to the open-ended questions deployed during the first phase of the study. The initial pool of items was rated by six different judges (raters) ${ }^{9}$ for content relevancy using a five point Likert scale. The raters also were asked to use a three point Likert scale for the clarity of each of the items. Three of the raters, who have taught the PHYS3313 and its subsequent PHYS3913 course, commented that most of the items that they validated were trivial or too easy. Only 47 items achieved consensual high ratings from experts and were deployed for field testing.

Figure 3 displays four items written for the PSCI. We will refer to these four items repeatedly throughout this paper. Although we are aware that multiple routes to obtain the correct answer are possible, we will exemplify the sequences of solving steps for two of these exemplary items.

Item 4.1 in Fig. 3(a) belongs to the category 4-Energy bands and requires the understanding of several definitions, i.e., atomic orbital and electronic state, then applying the definitions of the energy bands and band gap. It is based on a popular theoretical diagram illustrating the electronic energy bands versus the lattice constant of a crystalline solid $[53,54]$. Several students declared during the interviews that they were familiar with the knowledge content of the item 4.1 from their chemistry classes.

Item 6.1 in Fig. 3(b) was built to determine if previous instruction in a semiconductor devices course had occurred. It was also inspired by a popular textbook diagram [46,50]. The options for the item $\mathbf{6 . 1}$ are a succession of increasing levels of sophistication for the representation of an ideal semiconducting diode characteristic: option A represents the diode as a mechanical switch, option B introduces the opening threshold for an electronic-switch representation, option $\mathrm{C}$ adds the internal resistance of the diode, and option D emphasizes the nonlinear output of the semiconductor devices. During the interviews, students attending community colleges prior to enrolling in PHYS3313 said that they had chosen the options B or C for the item 6.1 because their experience with electronic circuits. Option A was chosen by students who claimed common knowledge. The later analysis of the data from field testing revealed that the item popularity for the item $\mathbf{6 . 1}$ was of $39 \%$ at preinstruction, but of a significantly higher $74 \%$ at postinstruction, irrelevant of the teaching method. We inferred that the item 6.1 can detect previous instruction in a semiconductor device course.

For item 8.2 in Fig. 3(c), the interviews revealed that the persons had difficulty interpreting an empty state as the complement event of an occupied state, at remembering level. The missing factual knowledge made this item written for application level more difficult than other items written for higher cognitive levels. The suggested solving

\footnotetext{
${ }^{9}$ The raters were self-selected from the expert participants in the PSCI study.
} 


\section{(a) Item 4.1)}

The figure below shows four possible distances between the closest neighboring atoms in a hypothetical atomic system. These distances are labeled $\mathbf{a}_{0}, \mathbf{a}_{1}, \mathbf{a}_{2}$ and $\mathbf{a}_{3}$. The energies for electrons of the atoms in the system are shown on the vertical axis. The energy difference

$\left(\mathbf{E}_{2}-\mathbf{E}_{1}\right)$ corresponds to ultraviolet light.

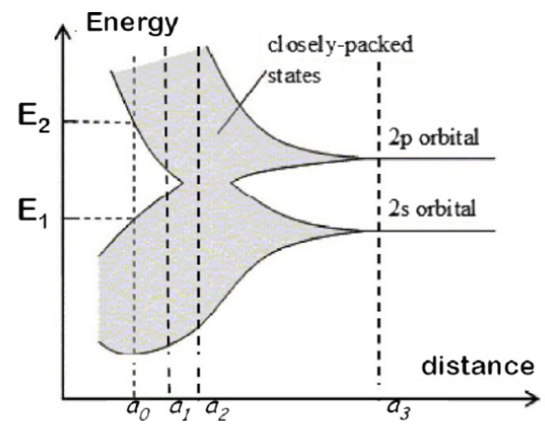

The diagram best illustrates the formation of
A. Energy bands.
B. Density of states
C. Direct band gap.
D. Orbital energy.

\section{(b) Item 6.1)}

Which of the following plots do you think best represents an ideal $\mathrm{P}-\mathrm{N}$ junction?
A. Plot A.
B. Plot B.
C. Plot C.
D. Plot D.
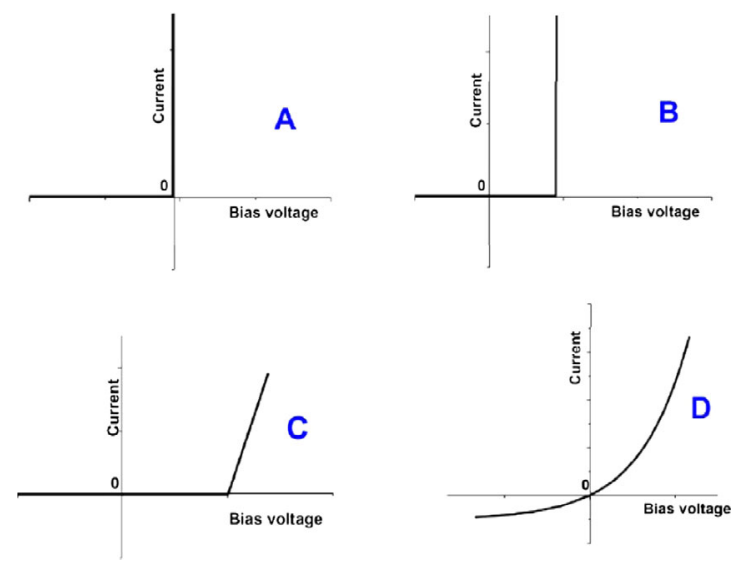

\section{(d) Item 7.2)}

An engineer tests the photoemission of a material XXX. Photoemission means the interaction of light with a material that can produce an electric current. The engineer mounts an ideal ammeter, able to detect even the tiniest electric current, as shown in the figure below The material XXX has equal sensitivity for blue and for green light. Blue light has higher frequency than green light. When the material XXX is illuminated with a $1 \mathrm{~mW}$ green laser beam, the ammeter reading is $0.01 \mathrm{~mA}$.

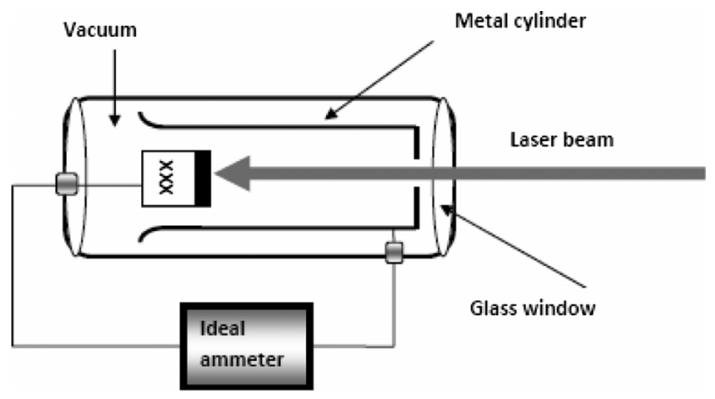

Which of the following statements best describes the ammeter response when the material XXX is illuminated with a $1 \mathrm{~mW}$ blue laser beam?
A. No change in the reading.
B. A zero value ammeter reading.
C. A non-zero value smaller than for the green laser reading.
D. A non-zero value greater than for the green laser reading.

FIG. 3. Four items in the PSCI. (a) An item at application level for the concept category 4-Energy Bands. The diagram for item 4.1 is an adaptation of the Figure 2.3.2 in Van Zeghbroeck's online textbook (Ref. [54]), (b) An item at application level for the concept category 6-PN junction $I-V$ plot, (c) An item at application level for the concept category 8-Statistics, (d) An item at evaluation level for the concept category 7-Quantum. The cartoon is inspired by the PhET interactive simulation for the "Photoelectric Effect" (Ref. [50]). All these items require procedural knowledge: to translate verbal constraints into mathematical language and to interpret diagrams and charts. 
sequence for item 8.2 is: remember (that the probability of occurrence of a range of events represents the area under the probability density function, that the total area equals 1 for a certain event, and that an empty state represents the complement event or the no-occurrence), understand (that only the area from 1 to $5 \mathrm{meV}$ contributes to the total probability, that probability is equally distributed on energy intervals, and that the probability of an energy interval equals the percentage of the corresponding area), apply (the definition of the probability of an energy interval to calculate a $25 \%$ chance that the interval from 4 to $5 \mathrm{meV}$ is occupied), then apply (the definition of the complement event to calculate the chance that a state in that interval is empty as $p=100 \%-25 \%=75 \%$ ).

Item 7.2 in Fig. 3(d) was initially developed for a class project that included interactive simulations [55]. A difficult step for solving item $\mathbf{7 . 2}$ was the connection at the analyze level between the pieces of information "same $1 \mathrm{~mW}$ " and "blue light has higher frequency than green light". Several interviewed students interpreted " $1 \mathrm{~mW}$ " as a measure of the photon number; this was a mistake at the understanding level, reflecting missing factual knowledge (knowledge of the units of measure). The suggested solving sequence for item 7.2 is: remember (the equation giving the photon energy as a function of frequency, the definitions of average power and average electric current, the mechanism of the photoelectric effect, and the fact that electrons carry electric charge), understand (that a green photon has enough energy to release an electron from the material XXX, that there is an equal probability to release an electron by either a green or a blue photon, that the $1 \mathrm{~mW}$ value means that the two laser beams have equal power, and that the total charge generated is directly proportional to the number of photoelectrons), apply (the photon energy formula to conclude that blue photons of higher frequency have greater energy than green photons; the definition of the photoelectric effect to conclude that the rate of the emitted electrons is proportional to the rate of incident photons; the information about equal probability of emission for green and blue to conclude that the rate of the incident photons determines uniquely the rate of emitted electrons; the average current formula to conclude that the ammeter reading is directly proportional to the number of electrons per unit time), analyze cause and effect implications (because constant sensitivity of the photomaterial, at equal photon rates there will be equal ammeter readings; because equal incident power, the photon rate for blue is lower than the photon rate for green), and, finally, evaluate that the lower photon rate for blue implies a smaller ammeter reading for blue than for green. Test takers may find shorter solution paths and guessing is yet another way to get to the correct answer.

\section{Participant selection}

The population targeted by our concept inventory is undergraduate students who are or have been enrolled in a semiconductor devices introductory course at a university using English language ${ }^{10}$ for instruction. Two categories of participants were involved in the PSCI development: undergraduate and graduate students whom we called "novices" and university professors whom we called "experts".

Participants enrolled on a volunteer basis. For snowball sampling the experts, we sent more than one hundred invitational e-mails to university professors teaching or conducting research in the field of semiconductors. The group of novices was recruited from 248 undergraduate students enrolled in the PHYS3313 class between Fall 2008 and Spring 2012, and from the 25 graduate students in the Department of Physics at OSU in Spring 2011 that had not taken a semiconductors course.

\section{Field testing}

Field testing was performed with 66 undergraduate and two graduate students at Oklahoma State University (OSU) between December 2010 and April 2012. The graduate students had not taken any prior physics of semiconductors course and sat only for preinstruction testing. Six former PHYS3313 students were sitting for the PSCI three or more semesters after finalizing the course.

In all the stages of the field testing, the PSCI was offered as an online quiz, available via a lock-down browser, in the proctored environment of a computer lab. At the beginning of each test administration, the novice participants were advised to answer to all the items according to their best knowledge. They were told that the PSCI test is under development and asked to report any lack of clarity of the problems. Backward navigation through pages was prevented and no feedback for either correctness of the answers or total score was provided after test administration.

A single attempt was allowed with the completion time restricted to one hundred minutes. This rather large time limit was set for possible slow-paced students. All participants finished before the time limit, with an average completion time of 1.5 min per question. The analysis of the time in testing revealed that two of the novices abandoned the testing after spending less than seven minutes for reading and submitting answers to only a few questions. The data from these two students were not retained in the sample for item calibration.

To assist the reader, Table I presents the details of the chronological development of the concept inventory. Table II gives the detailed composition of the sample in field testing. Thirty items (the PSCI-ALPHA version) were presented with insignificant variations of wording or of the order of the options in all the versions of the PSCI test. The analysis performed for a pooled sample of 83 responses showed that these 30 items satisfy the Rasch model.

\footnotetext{
${ }^{10} \mathrm{We}$ do not know if a translated version of the PSCI items would keep the current parametrization.
} 
TABLE I. Chronological development of the PSCI and schedule for test administration.

\begin{tabular}{lcccl}
\hline \hline PSCI test version & Number of items & Date & Persons & Comment \\
\hline Open-ended questions deployed & $>100$ & Aug 2009 & 72 & 16 experts, 56 novices \\
PILOT 1 & 47 & Dec 2010 & 14 & Novices, post-test \\
Interviews conducted & & Dec 2010 & 10 & 5 experts, 5 novices \\
PILOT 2 & 45 & Jan 2011 & 5 & Novices, pretest \\
PILOT 2 & 45 & April 2011 & 11 & Novices, post-test \\
Interviews conducted & & April 2011 & 8 & Novices \\
PILOT 2 & 45 & Aug 2011 & 12 & Novices, pretest \\
PILOT 2 & 45 & Dec 2011 & 7 & Novices, post-test \\
Interviews conducted & & Dec 2011 & 1 & Novice \\
ALPHA & 30 & Jan 2012 & 10 & Novices, pretest \\
ALPHA & 30 & Apr 2012 & 24 & Novices, post-test \\
BETA & 18 & Apr 2013 & 35 & Independent sample of novices, post-test \\
\hline \hline
\end{tabular}

TABLE II. Person sample for the PSCI item calibration.

\begin{tabular}{llc}
\hline \hline Criterion & \multicolumn{1}{c}{ Categories } & Counts \\
\hline Instruction & PRE & 27 \\
in PHYS3313 & POST & 56 \\
Lecture format & traditional & 31 \\
(for POST only) & non-traditional & 25 \\
Took the test & once & 45 \\
& twice & $19^{\mathrm{c}}$ \\
University classification & undergraduate $^{\mathrm{a}}$ & 60 \\
Graduate & 2 \\
Gender & alumni $^{\mathrm{b}}$ & 2 \\
First language & female & 5 \\
& male & 59 \\
& English & 48 \\
\hline \hline
\end{tabular}

\footnotetext{
ain PRE only.

${ }^{b}$ in POST only.

${ }^{c}$ all these 19 repeated measures students took the PHYS3313 in traditional lecture format.
}

\section{CALIBRATION AND MEASUREMENTS}

\section{A. Classical analysis}

The information collected from the PSCI field testing was separated into item-data and person-data. The itemdata were examined for distracter effectiveness and item functioning. Table III is an excerpt of the answer matrix collected during the PSCI field testing. Each row represents the answer pattern of a person and each column stores the answers for one item. An answer pattern is the sequence of item scores for a person. The item score can be 1 for correct, 0 for incorrect, or NA for not submitted. The person answer patterns were examined to determine whether there is any significant sample split in field testing.

The matrix retained for item calibration contained the answer patterns from 27 novices at pre- and 56 at post-test; 19 students took the test both before and after their PHYS3313 course. Although the proportion of correct answers was greater after instruction than before
TABLE III. Excerpt from answer matrix. Note that C03, E01, and F03 are codes for persons. 1.2, 2.2, etc., are codes for PSCI items.

\begin{tabular}{lcccccccccc}
\hline \hline & \multicolumn{10}{c}{ PSCI items } \\
\cline { 2 - 11 } Person & 1.2 & 2.2 & 2.3 & 2.4 & 2.6 & 3.2 & 4.1 & 4.2 & 4.3 & 4.4 \\
\hline C03 & 0 & 1 & 0 & 1 & 0 & 0 & 1 & 0 & 1 & 1 \\
E01 & 1 & 1 & 1 & 1 & 1 & 1 & 1 & 0 & 1 & 1 \\
F03 & 1 & 0 & 1 & 0 & 0 & 0 & 0 & 0 & 0 & 0 \\
\hline \hline
\end{tabular}

instruction, there was no significant splitting of the answer matrix into two categories of respondents. As the test scores were not normally distributed, we employed Fisher's exact test for comparing the score frequencies of the 19 test repeaters and of the one-time test takers separately for preinstruction and for postinstruction. No significant difference was found ( $p$-values greater than 0.10 ). We also found that for the majority of the incorrect answers at postinstruction the test repeaters did not choose the same option as at preinstruction. Consequently, for item calibration purposes, we treated the answer patterns of the 19 test repeaters as independent measures. This was not an unusual decision because of the four month time interval between the pre- and post-test. It followed the precedent of the research group developing the BEMA concept inventory [56,57].

Linear discriminant analysis and exact Fisher's tests were employed for identifying a possible group split due to demographic factors such as gender, first language, or number of semesters since taking the PHYS3313 course. No significant difference due to the demographics was detected. We concluded that we had just one population in field testing.

The answer patterns were weakly correlated to each other, with an overall item-item correlation ${ }^{11}$ of 0.06 . This

\footnotetext{
${ }^{11}$ The reader can find the classical correlation coefficients for the various sets of items discussed in this paper in the Supplemental Material [15].
} 


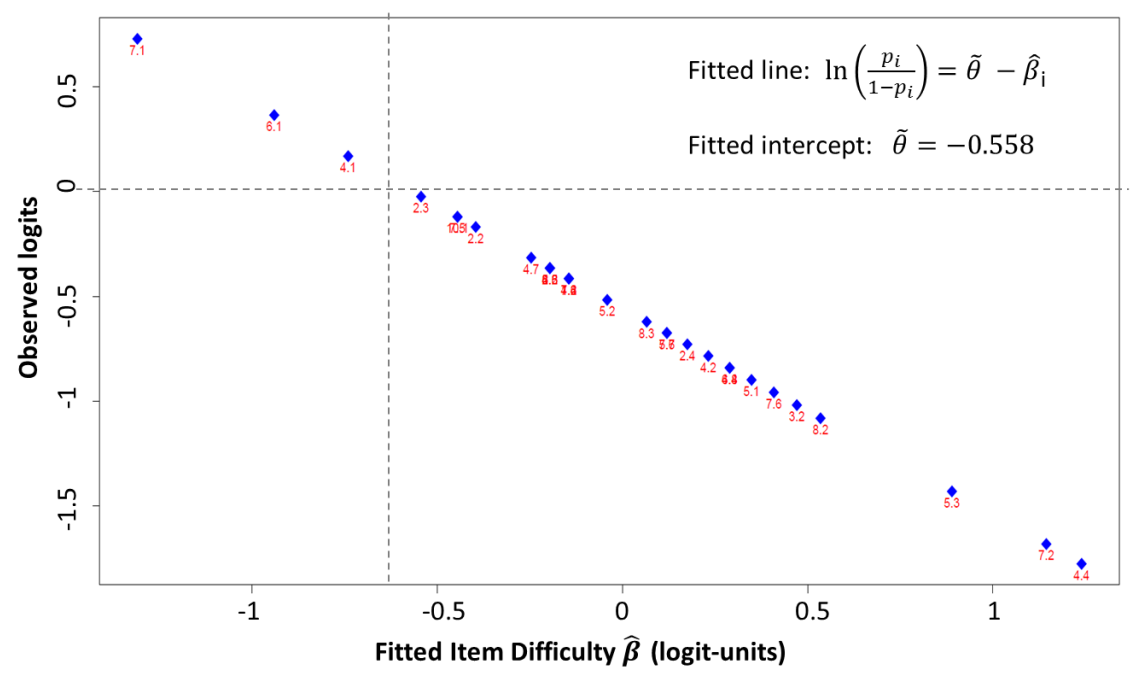

FIG. 4. Rasch calibration of the items in PSCI-ALPHA. Thirty items in field testing fit the Rasch unidimensional model. The observed logits fall on a -1 slope line. The horizontal dashed line represents the empirical zero-logit level for the $50 \%$ item popularity. The vertical dashed line marks the intercept with the empirical zero-logit level, at $\tilde{\theta}=-0.558$ logit, and suggests that the persons in field testing had, on average, less than a $50 \%$ chance to solve correctly the entire 30 -item set.

supported the local independence claim for most of the items in PSCI and the pursuit of a one-dimensional Rasch calibration. Several groups of items were intentionally written to be very similar in content and to cover the same concept. We found that the item-item correlation coefficients of the similar items were significantly greater than the average. The maximum correlation coefficient was of 0.505 for the similar items $\mathbf{5 . 2}$ and $\mathbf{5 . 6}$ and the minimum was of 0.013 for the dissimilar items $\mathbf{8 . 2}$ and 5.2.

The first eigenvalue of the variance-covariance matrix was twice the value of the next highest eigenvalue. The existence of a major underlying factor that could explain the data variance represented classical evidence that the test has one principal dimension and justified the pursuit of a linear model. The 0.82 value of the coefficient Guttman's lambda-6 [58] implied the test reliability in classical interpretation.

\section{B. CML Rasch modeling results}

\section{Goodness-of-fit and item parameter estimation}

Because our prime interest was to generate an invariant item scale, we chose the open-source $\mathrm{R}^{12} \mathrm{eRm}$ package for response data modeling [59]. This $e R m$ package implements CML algorithms that do not include persons' abilities in the item parameters estimation [32]. Thirty PSCI items satisfied the Rasch model and were retained under the name of PSCI-ALPHA. The items deployed in

\footnotetext{
${ }^{12} \mathrm{R}$ is a language and environment for statistical computing and graphics. It is a GNU project.
}

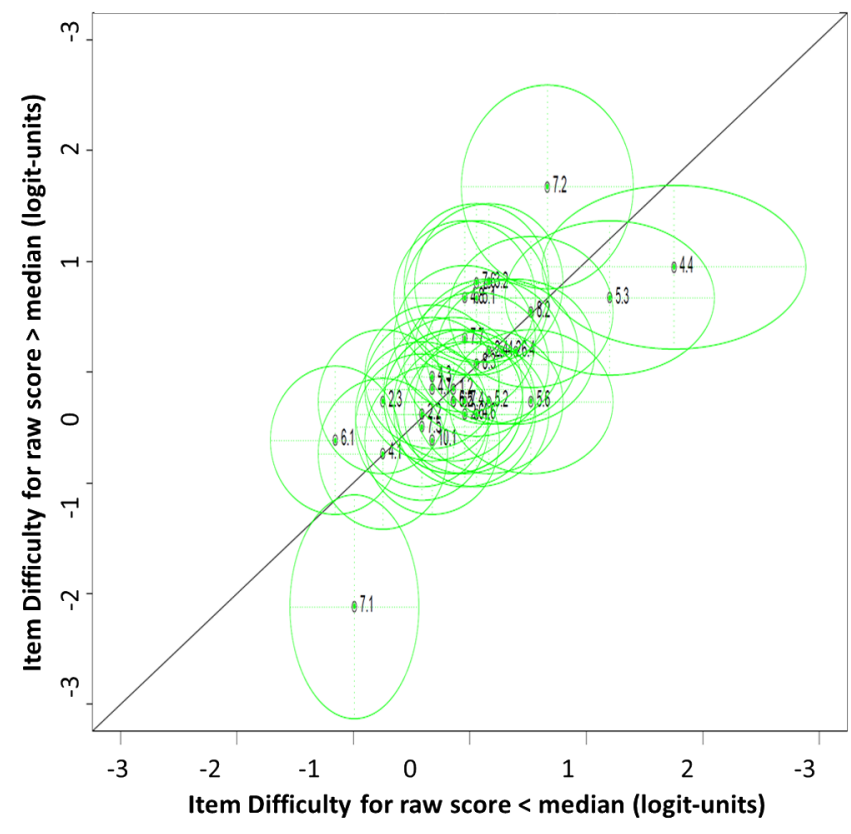

FIG. 5. Likelihood ratio goodness-of-fit with median-split criterion for PSCI-ALPHA. In order to check the invariance of the Rasch scale, the item parameters are plotted for two subgroups of the sample in field testing. The item parameters estimated from the answer patterns of 41 persons with raw scores lower than median are plotted on the horizontal axis. The item parameters estimated for 41 persons with scores higher than the median are plotted on the vertical axis. The two 95\% confidence intervals of each double-estimated parameter give an ellipse. The items invariant to sample split fall on or close to the bisector of slope 1 shown as a solid black line. The items situated farthest from the bisector have the poorest fit. 
TABLE IV. Item fit statistics for the CML Rasch calibrated PSCI-ALPHA. MSQ = mean square, Outfit MSQ = the sum of the squared standardized residuals averaged over all the persons taking the test, Infit MSQ = the weighted average of the squares of the residuals (it must be close to 1 for a good fit), Outfit- $t=$ chi-square-like statistic for Outfit MSQ, Infit- $t=$ chi-square-like statistic for Infit MSQ.

\begin{tabular}{|c|c|c|c|c|c|c|c|c|c|}
\hline Item & Outfit MSQ & Infit MSQ & Outfit- $t$ & Infit- $t$ & Item & Outfit MSQ & Infit MSQ & Outfit- $t$ & Infit- $t$ \\
\hline $1.2^{\mathrm{a}}$ & 1.047 & 1.041 & 0.96 & 0.92 & $5.3^{\mathrm{a}}$ & 1.014 & 0.973 & 0.18 & -0.2 \\
\hline $2.2^{\mathrm{a}}$ & 1.079 & 1.064 & 1.99 & 1.74 & $5.5^{\mathrm{a}}$ & 0.955 & 0.965 & -0.93 & -0.8 \\
\hline 2.3 & 1.075 & 1.067 & 2.01 & 1.94 & $5.6^{\mathrm{a}}$ & 0.915 & 0.919 & -1.27 & -1.42 \\
\hline $2.4^{\mathrm{a}}$ & 0.949 & 0.959 & -0.69 & -0.65 & $6.1^{\mathrm{a}}$ & 1.09 & 1.066 & 1.93 & 1.65 \\
\hline 2.6 & 0.924 & 0.939 & -1.61 & -1.42 & $6.2^{\mathrm{a}}$ & 0.961 & 0.959 & -0.81 & -0.96 \\
\hline 3.2 & 1.055 & 1.05 & 0.63 & 0.67 & 6.4 & 0.98 & 0.993 & -0.22 & -0.07 \\
\hline $4.1^{\mathrm{a}}$ & 0.948 & 0.953 & -1.35 & -1.34 & 7.1 & 0.799 & 0.842 & -3.06 & -2.86 \\
\hline 4.2 & 1.047 & 1.033 & 0.66 & 0.55 & $7.2^{\mathrm{a}}$ & 1.171 & 1.065 & 1.14 & 0.55 \\
\hline 4.3 & 1.033 & 1.023 & 0.72 & 0.56 & 7.4 & 0.946 & 0.953 & -1.07 & -1.05 \\
\hline 4.4 & 0.849 & 0.919 & -0.86 & -0.52 & $7.5^{\mathrm{a}}$ & 1.007 & 1.005 & 0.19 & 0.17 \\
\hline $4.6^{\mathrm{a}}$ & 0.967 & 0.96 & -0.64 & -0.88 & $7.6^{\mathrm{a}}$ & 1.054 & 1.041 & 0.65 & 0.58 \\
\hline $4.7^{\mathrm{a}}$ & 0.981 & 0.983 & -0.39 & -0.4 & $7.7^{\mathrm{a}}$ & 1.01 & 1 & 0.19 & 0.03 \\
\hline $4.8^{\mathrm{a}}$ & 1.133 & 1.073 & 1.67 & 1.11 & $8.2^{\mathrm{a}}$ & 0.967 & 0.972 & -0.29 & -0.29 \\
\hline 5.1 & 1.129 & 1.089 & 1.54 & 1.26 & $8.3^{\mathrm{a}}$ & 1.026 & 1.024 & 0.44 & 0.46 \\
\hline 5.2 & 0.937 & 0.932 & -1.12 & -1.38 & 10.1 & 0.966 & 0.967 & -0.87 & -0.92 \\
\hline
\end{tabular}

ancluded in the 18-item selection PSCI-BETA.

field testing with no fit on the Rasch scale were quarantined for possible refurbishing and reusing. We will show how Rasch scaling allowed us to separate the items in subscales and thus to track directly knowledge and knowledgecoherence.

The observed logits of the items in PSCI-ALPHA are plotted against the fitted item parameters in Fig. 4. The easiest item in PSCI-ALPHA is $\mathbf{7 . 1}$ with an estimate of $\beta[7.1]=-1.31 \pm 0.232$. The most difficult is $\mathbf{4 . 4}$ with an estimate of $\beta[4.4]=1.24 \pm 0.306$. The $x$ intercept of the plot in Fig. 4 gives a rough estimate of the median ability of the persons in field testing, $\tilde{\theta}=-0.558$. The negative value of this intercept signals that the 30-item PSCI-ALPHA set is, on average, too difficult for the average person in field testing.

A series of likelihood ratio goodness-of-fit tests [60] confirmed that the answers to the items in the PSCIALPHA test are invariant across distinct subgroups in field testing. Figure 5 illustrates the item parameter invariance if the group split criterion is the median raw score. The items that have equal estimated parameters for the two split groups lie on the invariance line of slope 1 . The extreme items 7.1 and 4.4 are the furthest from the invariance line.

The informal parameters outfit mean squares and infit mean squares [34] were employed for further checking the goodness-of-fit of the model. The outfit mean square statistic, which measures the average mismatch between data and model, was useful to identify outliers in the sample of persons in field testing. The infit mean square and the approximate $t$-statistic Infit- $t$ [61] helped identify those items generating outliers and bias. The usual requirement for an unbiased Rasch scale is that $\mid$ Infit- $t \mid \leq 2[62,63]$. The goodness-of-fit results for Rasch calibration are shown in
Table IV. One should note that only one item in PSCIALPHA, 7.1, had an $\mid$ Infit- $t \mid$ statistic greater than 2. The Rasch parametrization of the 30 items in PSCI ALPHA is given in Table V.

When solving problems, mistakes can be generated by missing pieces of knowledge and by the lack of training with organizing and matching pieces of information. We believe that the attribute "item difficulty" reflects the combination of expert quality knowledge and level of the cognitive processes involved in answering that item. Our items written for the same cognitive process have different difficulties because they address different concepts, require different factual and procedural knowledge, and have different numbers of solving steps. Let us look at the items 4.1 and 8.2 from the subset application. The item 4.1 [in Fig. 3(a)] asks to apply definitions from the class's textbook to a diagram in a two-step solution. It has a low difficulty $\beta[4.1]=-0.741$ logit. The item 8.2 [in Fig. 3(c)] asks to apply three or more definitions from both physics and statistics and may take more than two steps for solving. It has the $\beta[8.2]=0.508$ difficulty and is located at more than one logit to the right of the item 4.1. ${ }^{13}$ An item attributed to the superior cognitive level evaluation, such as 1.2, is easier than an item attributed to the lower cognitive level application, such as $\mathbf{8 . 2}$, partially because the average person in field testing was more familiar with the knowledge content of the former than of the latter.

\footnotetext{
${ }^{13}$ The students of the Spring 2013 PHYS3313 class perceived the item 4.1 more difficult than its calibrated value. For this group of students, the items $\mathbf{4 . 1}$ and $\mathbf{8 . 2}$ had roughly equal popularities. We inferred that these students were unfamiliar with the concept of energy band formation.
} 
The good fit of the 30 items on the Rasch onedimensional scale supports the idea that there is a unique construct measured by the PSCI-ALPHA. We named this common trait as "composite ability to operate with basic conceptual knowledge of physics of semiconductors". The novice participants interviewed after the postinstruction testing perceived the "knowledge of inferring information from graphs and plots" as an important procedural knowledge component of the PSCI-ALPHA.

The items retained in the PSCI-ALPHA address nine of the initial ten concept categories. The categories 1-Diffusion and 10-Crystals had one surviving item each. For analysis purposes, we created a merged category $1 \& \mathbf{2}$ Carrier transport to include items from 1-Diffusion and 2-Drift. We checked if the items written for each concept category do indeed form subscales on the PSCI-ALPHA by employing a different IRT approach, which is described in Sec. IV C.

\section{Person parameter estimation with the 30-item PSCI-ALPHA}

The CML algorithm gives the item parameters independent of the distribution of the persons taking the test. The fitted item parameters are then substituted back into the algorithm to estimate the person parameters according to Eq. (4). Those persons with incomplete answers have their ability estimated separately, by interpolation. The abilityparameters of the persons submitting answers to all the 30 items and the standard errors are tabulated in Table VI. Table VII shows the result of interpolation versus direct estimation for pairs of participants with the same raw score. In Fig. 6, the item parameters and the person parameters are plotted on the same scale. The median of the estimated person parameters in field testing lies to the left of the median item difficulty at $-0.585 \pm 0.391$. This implies that the average person in field testing had between $27.4 \%$ and $45.2 \%$ probability to solve correctly the entire PSCI-ALPHA.

The Rasch calibrated PSCI-ALPHA instrument locates a person within a 0.802 -logit average sized $95 \%$ confidence interval. The standard errors for the sample in field testing are consistent with the theoretical average value of 0.447 logit for a 30-item instrument [64]. The ability spread from -1.47 to 0.73 logit reflects the variability of the sample in field testing.

Although we did not obtain a sharp discrimination between person abilities, the crucial benefit of the Rasch calibrated scale is that persons are not compared among themselves but with fixed located items. Our main goal of comparing student populations before and after instruction could still be achieved if the PSCI test could assign students to distinct proficiency categories. The following sections will show how.

The score-to-ability conversion function resulting from the Rasch calibration can predict person parameters for
TABLE V. CML Rasch item parametrization for PSCI-ALPHA. Item subsets can be extracted for quick diagnostic. Each item subset is a Rasch invariant scale. Items are listed in the ascending order of their difficulty. Std. Error=standard error of the estimation; $I-V$ plot, application, evaluation, quantum, transport, band are the names of the six pairs of items in the subset pairs.

\begin{tabular}{|c|c|c|c|c|}
\hline \multirow[b]{2}{*}{$\begin{array}{l}\text { Item } \\
\text { label }\end{array}$} & \multirow[b]{2}{*}{$\begin{array}{c}\text { Item } \\
\text { parameter }\end{array}$} & \multirow[b]{2}{*}{$\begin{array}{l}\text { Standard } \\
\text { error }\end{array}$} & \multicolumn{2}{|c|}{ Subscales including this item } \\
\hline & & & Bloom's-level & PAIRS \\
\hline 7.1 & -1.310 & 0.232 & & \\
\hline 6.1 & -0.940 & 0.222 & Application & $I-V$ plot \\
\hline 4.1 & -0.741 & 0.219 & Application & Application \\
\hline 2.3 & -0.544 & 0.219 & & \\
\hline 7.5 & -0.446 & 0.219 & Analysis & Quantum \\
\hline 10.1 & -0.446 & 0.219 & & \\
\hline 2.2 & -0.396 & 0.219 & Analysis & \\
\hline 4.7 & -0.246 & 0.221 & Application & \\
\hline 2.6 & -0.196 & 0.222 & & \\
\hline 4.3 & -0.196 & 0.222 & & \\
\hline 5.5 & -0.196 & 0.222 & Application & \\
\hline 6.2 & -0.196 & 0.222 & Analysis & $I-V$ plot \\
\hline 1.2 & -0.145 & 0.223 & Evaluation & Transport \\
\hline 4.6 & -0.145 & 0.223 & Analysis & Bands \\
\hline 7.4 & -0.145 & 0.223 & & \\
\hline 5.2 & -0.041 & 0.226 & & \\
\hline 8.3 & 0.065 & 0.229 & Evaluation & Evaluation \\
\hline 5.6 & 0.120 & 0.231 & Analysis & \\
\hline 7.7 & 0.120 & 0.231 & Analysis & Quantum \\
\hline 2.4 & 0.175 & 0.233 & Application & Transport \\
\hline 4.2 & 0.232 & 0.235 & & \\
\hline 4.8 & 0.289 & 0.237 & Analysis & Bands \\
\hline 6.4 & 0.289 & 0.237 & & \\
\hline 5.1 & 0.348 & 0.240 & & \\
\hline 7.6 & 0.409 & 0.243 & Evaluation & Evaluation \\
\hline 3.2 & 0.471 & 0.246 & & \\
\hline 8.2 & 0.535 & 0.250 & Application & Application \\
\hline 5.3 & 0.890 & 0.274 & Evaluation & \\
\hline 7.2 & 1.144 & 0.297 & Evaluation & \\
\hline 4.4 & 1.240 & 0.306 & & \\
\hline
\end{tabular}

scores not yet observed. This represents a major benefit of the Rasch calibration. Therefore, the Rasch calibrated PSCI-ALPHA test can be employed to measure the basic knowledge of physics of semiconductors, for groups of persons with a lower or higher ability than the sample in field testing.

\section{The PSCI-BETA Instrument}

Following the PSCI-ALPHA calibration, we needed a supplementary sample of students for verifying the item parametrization stability. Our colleague, the instructor for the PHYS3313 class of the Spring 2013 semester, agreed to offer the PSCI test during his final review lecture. Test administration time constraint was a reason to select only a portion of the 30-item PSCI-ALPHA for this 
TABLE VI. Person parameters for the sample in field testing; CML Rasch estimation. Values valid only for the persons submitting answers to all questions (NA Group1).

\begin{tabular}{|c|c|c|c|c|c|}
\hline \multicolumn{3}{|c|}{ PSCI-ALPHA } & \multicolumn{3}{|c|}{ PSCI-BETA } \\
\hline Raw score & Ability estimate & Standard error & Raw score & Ability estimate & Standard error \\
\hline 0 & -2.73482 & NA & 0 & -3.19284 & NA \\
\hline 1 & -2.52425 & NA & 1 & -2.68322 & NA \\
\hline 2 & -2.31367 & NA & 2 & -2.1736 & 0.758574 \\
\hline 3 & -2.10310 & NA & 3 & -1.69067 & 0.642548 \\
\hline 4 & -1.89253 & NA & 4 & -1.32113 & 0.578156 \\
\hline 5 & -1.68195 & NA & 5 & -1.01102 & 0.538301 \\
\hline 6 & -1.47138 & 0.46695 & 6 & -0.73578 & 0.51274 \\
\hline 7 & -1.26504 & 0.44258 & 7 & -0.48167 & 0.496763 \\
\hline 8 & -1.07758 & 0.42410 & 8 & -0.23973 & 0.48802 \\
\hline 9 & -0.90392 & 0.40991 & 9 & -0.00333 & 0.485382 \\
\hline 10 & -0.74050 & 0.39902 & 10 & 0.233306 & 0.488507 \\
\hline 11 & -0.58471 & 0.39077 & 11 & 0.475954 & 0.497726 \\
\hline 12 & -0.43448 & 0.38473 & 12 & 0.731257 & 0.514154 \\
\hline 13 & -0.28816 & 0.38061 & 13 & 0.98954 & NA \\
\hline 14 & -0.14431 & 0.37823 & 14 & 1.247824 & NA \\
\hline 15 & -0.00162 & 0.37749 & 15 & 1.506107 & NA \\
\hline 16 & 0.14111 & 0.37835 & 16 & 1.76439 & NA \\
\hline 17 & 0.28509 & 0.38085 & 17 & 2.022674 & NA \\
\hline 18 & 0.43164 & 0.38508 & 18 & 2.280957 & NA \\
\hline 19 & 0.57878 & NA & & & \\
\hline 20 & 0.72592 & NA & & & \\
\hline 21 & 0.87306 & NA & & & \\
\hline 22 & 1.02020 & NA & & & \\
\hline 23 & 1.16734 & NA & & & \\
\hline 24 & 1.31448 & NA & & & \\
\hline 25 & 1.46162 & NA & & & \\
\hline 26 & 1.60876 & NA & & & \\
\hline 27 & 1.75590 & NA & & & \\
\hline 28 & 1.90304 & NA & & & \\
\hline 29 & 2.05018 & NA & & & \\
\hline 30 & 2.19732 & NA & & & \\
\hline
\end{tabular}

postinstruction diagnostic. As discussed in Sec. II B, one property of a Rasch scale is that any selection of calibrated items is yet another Rasch scale.

We selected 18 items from the PSCI-ALPHA for a testlet that we labeled PSCI-BETA. These 18 items cover the concepts $1 \&$ 2-Carrier transport, 4-Energy bands, 5-Carrier concentration, 6-PN junction $I-V$ plot, 7-Quantum

TABLE VII. Person parameters for equal scores but different number of answers submitted. NA Group=all persons not submitting answers for the same items, NA Group1=persons with all answers submitted, NA Group2=persons not submitting an answer for item 8.2, NA Group4=persons not submitting an answer for item $\mathbf{4 . 4}$.

\begin{tabular}{lccc}
\hline \hline Raw Score & NA group & Ability estimate & Standard error \\
\hline 11 & 1 & -0.65419 & 0.41648 \\
11 & 4 & -0.64268 & 0.41827 \\
12 & 1 & -0.48334 & 0.41049 \\
12 & 2 & -0.43519 & 0.41629 \\
\hline \hline
\end{tabular}

approach, and 8-Statistics. The PSCI-BETA and the PSCIALPHA are both centered on a zero difficulty, as one can verify by averaging the item parameters listed in Table V. Because the easiest and the hardest items were not included, the range of the PSCI-BETA is 0.5 logit shorter than its parent set. Likewise, the 18-item set has less internal variability than its parent because the magnitudes of its Infit- $t$ statistics are less than 2 .

We recalculated the abilities of the persons in field testing via the PSCI-BETA set and found them stable within a standard error with the exception ${ }^{14}$ of the initial raw score of 7 . A consequence of reducing the number of items in the test was the increase of the standard error of estimation with 0.11 -logit at the center and with 0.33 logit at the extremity of the ability scale.

\footnotetext{
${ }^{14}$ As a score of 7 represents a maximum for binomial guessing in 30 trials, and as low scores are usually affected by guessing, we attributed the change in ability estimation to guessing the answers for some items present in the ALPHA but not in BETA.
} 


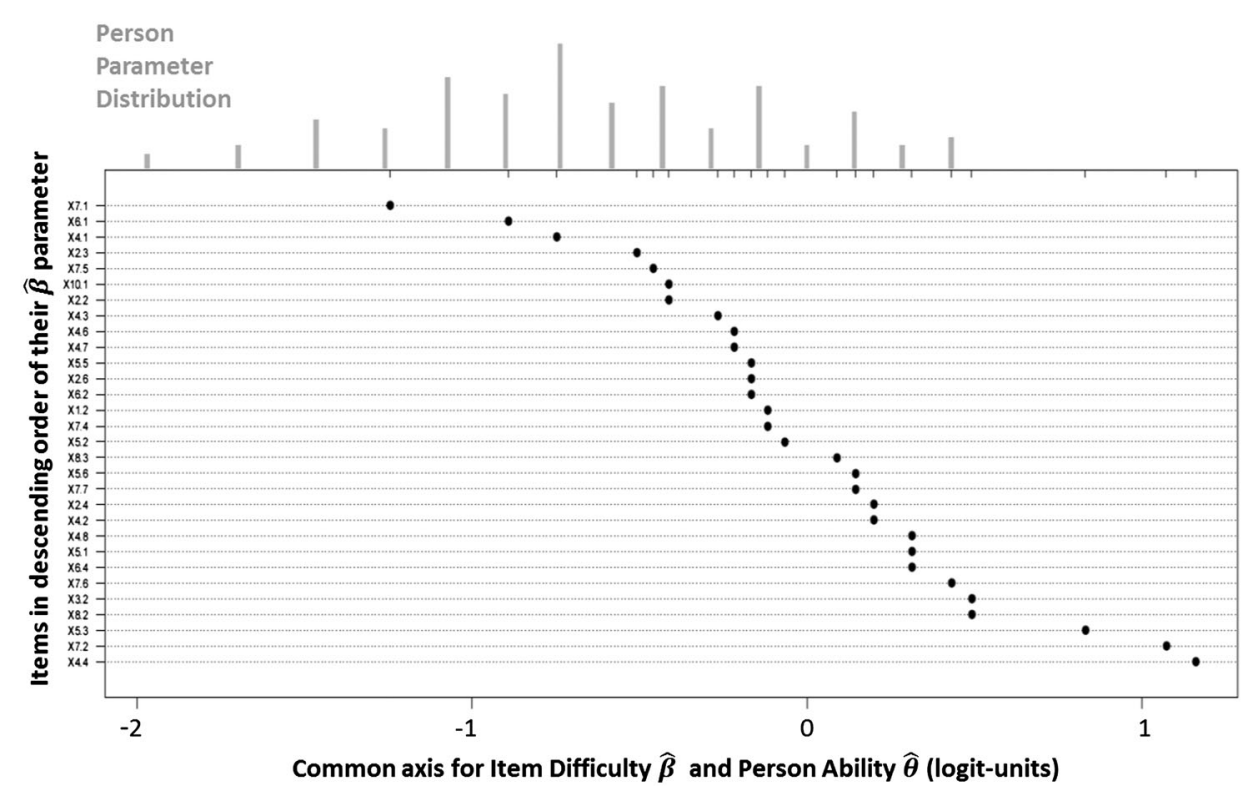

FIG. 6. Person-Item map for PSCI-ALPHA. The black dots represent item locations. Each black dot corresponds to a tick on the upper horizontal axis. The gray bars at the top mark person locations $\hat{\theta}_{\nu}$ on the common logit axis. The height of a gray bar is proportional to the number of persons at that location (histogram). The 19 persons repeating the test have two distinct estimated abilities, at pre- and postinstruction; therefore, a total of $64+19$ persons are depicted in the histogram person parameter distribution. The median ability is $-0.585 \pm 0.391$ logit, below the 0 logit of the test difficulty. The mode of the ability distribution is $-0.741 \pm 0.399$ logit.

In order to offset the greater uncertainty in person location due to the coarser 18-item scale, we thought of a triangulation method. We split the PSCI-BETA into three separate subscales, each meeting the invariance requirement. We had enough calibrated items for building subsets at the application, analysis, and evaluation cognitive levels but not enough for building a subset for the understandinglevel. These three subsets would later serve as anchors for linking new items to the existing ones and obtaining an item bank [65]. Their characteristics are listed in Table VIII. The subset Application is more than one standard error easier, the subset Analysis has practically the same average difficulty, and the subset Evaluation has a larger average difficulty than the full scale PSCI-ALPHA.

We constructed a fourth subscale labeled PAIRS. It contains pairs of items that share common elements, i.e., same concept or same cognitive level. The items in PAIRS were fit with the partial credit model [66] and constitute a link between the other three subsets. The person parameter

TABLE VIII. Subscales in the CML Rasch calibrated PSCI test. Uncertainty $=$ average standard error of the item location.

\begin{tabular}{lcccc}
\hline \hline & & \multicolumn{3}{c}{ Characteristics, in logit units } \\
\cline { 3 - 5 } Item set & Item count & Center & Range & Uncertainty \\
\hline ALPHA & 30 & 0 & 2.550 & 0.2340 \\
BETA & 18 & 0.0164 & 2.084 & 0.2342 \\
Application & 6 & -0.2355 & 1.475 & 0.2278 \\
Analysis & 7 & -0.0934 & 0.735 & 0.2260 \\
Evaluation & 5 & 0.4726 & 1.289 & 0.2532 \\
\hline \hline
\end{tabular}

estimated via PAIRS should provide information about the consistency of the answers, therefore of one's knowledgecoherence.

The 18-item set and its four subscales to be employed for knowledge diagnostics were labeled the PSCI-BETAInstrument (PSCI-BETA-I). The internal structure of the PSCI-BETA-I is pictured in Fig. 7. The PSCI-BETA-I offers five different ability estimations that are combined for drawing person profiles. The subscales in the

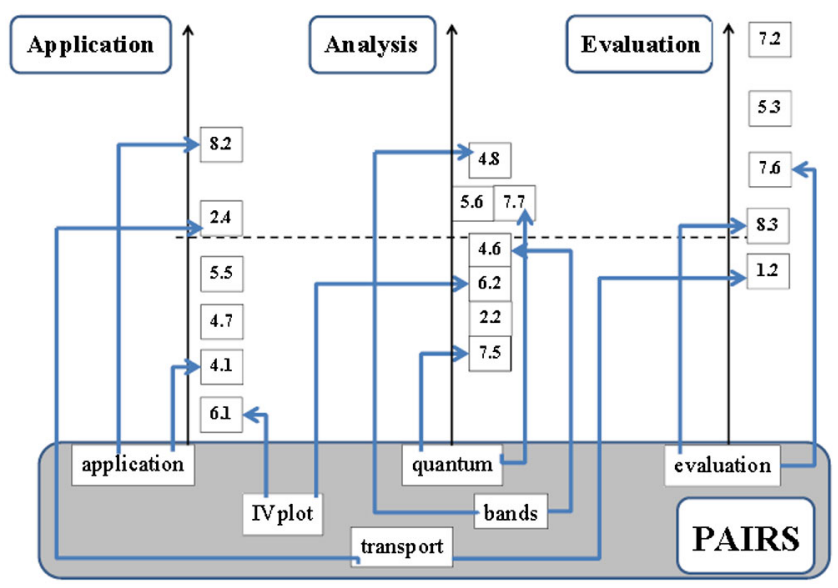

FIG. 7. Subsets in the PSCI-BETA-Instrument. The items in each subset are represented in the increasing order of their difficulty. The horizontal dashed line stands for the zero-logit median difficulty. The shaded box PAIRS contains the labels of the six pairs of items. The items paired together are indicated by arrows starting from that pair's label in the shaded box. 


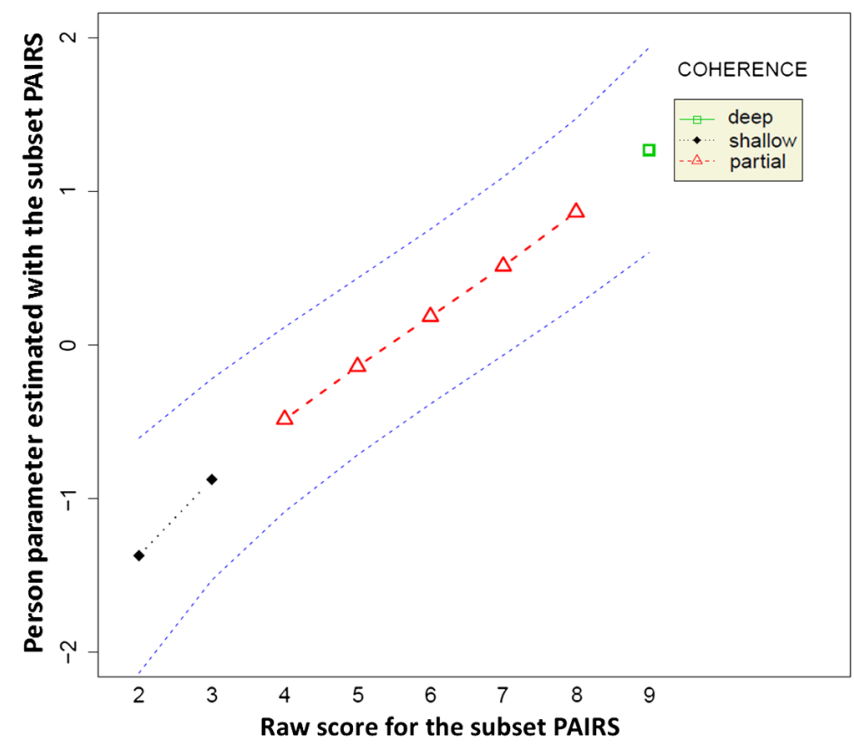

FIG. 8. Assignment to a knowledge-coherence category. The person parameters estimated in the partial credit model are plotted against the raw scores for the item-subset PAIRS. The maximum possible score is 12 . The dashed lines are at 1 standard error distance from the point estimate. The assignment to one of the three knowledge-coherence categories listed in the legend depends on the person's location on the scale PAIRS.

PSCI-BETA-I are useful for scaling persons within the same sample. These subscales can diagnose missing knowledge or lack of knowledge-coherence, which is the crucial information that an instructor needs before tailoring learning activities or revisiting concepts one week before a class exam. We demonstrate how to employ the PSCI-BETA for diagnostic at post-instruction in the following section.

\section{Ability estimation with the PSCI-BETA Instrument}

The 35-student independent sample to be diagnosed by the PSCI-BETA-I at postinstruction in Spring 2013 was from a traditional lecture format course. We recalculated the item parametrization with the new data and found stability with the exception of item 4.1 at the application level. The item 4.1 was perceived with 1.3 logit more difficult than the calibrated value. We inferred that the students in Spring 2013 were unfamiliar with the energy band formation.

The person parameter estimated by the subset PAIRS served to assign the answer patterns to three categories of knowledge-coherence: "partial" for persons within two standard errors from the zero-logit median, "shallow" for persons at more than 2 standard errors from origin to the negative direction, and "deep" for persons at more than 2 standard errors from origin to the positive direction. The coherence category assignment and its relationship to the observed score for the 12-item set pairs are illustrated by Fig. 8 .

The instructor of the PHYS3313 class of Spring 2013 received the results of the PSCI-BETA-I measurement in the form of two visual charts. The quick diagnostic chart (Fig. 9) informed the instructor that one student was located above and seven were located below the median of the item scale. The student above the median had a deep understanding of the concepts addressed by the problems that she

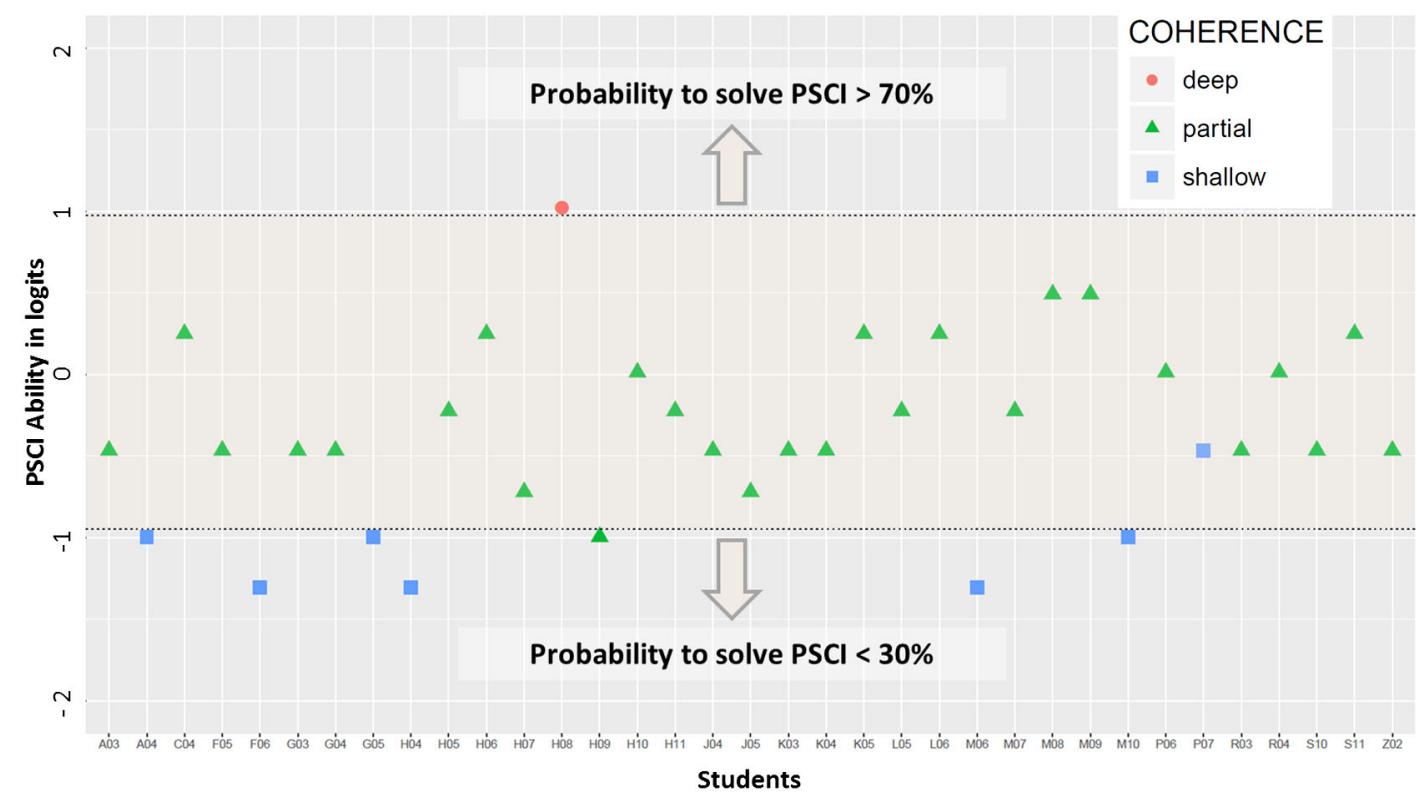

FIG. 9. Quick diagnostic chart. The name codes of the students in postinstruction testing are listed on the horizontal axis. Each student is represented by a symbol. The shape and color of the symbols denote the knowledge-coherence category. The vertical coordinate gives the PSCI-estimated ability parameter $\hat{\boldsymbol{\theta}}$. The highlighted band represents the $95 \%$ confidence interval of a zero (median) ability. The persons located below the highlighted band have less than $30 \%$ chance to solve correctly the entire PSCI-BETA. The persons located above the highlighted band have a chance greater than $70 \%$ to solve correctly the test. 


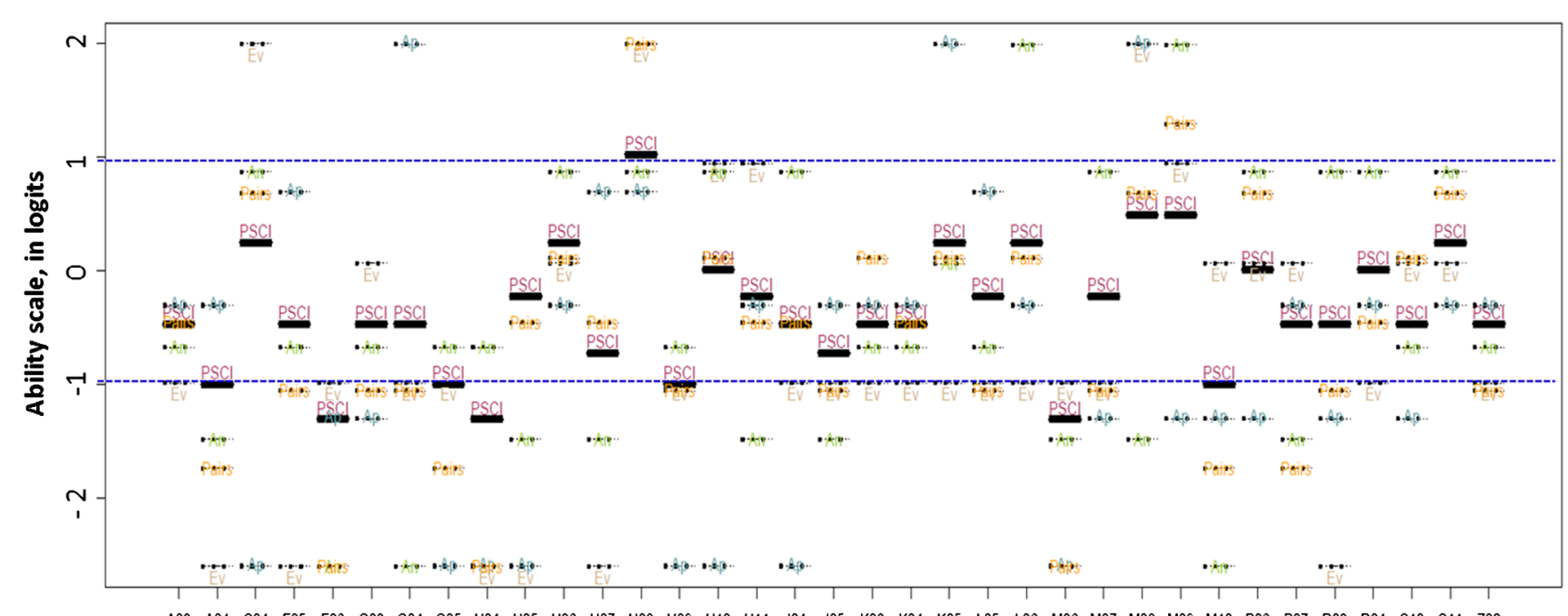

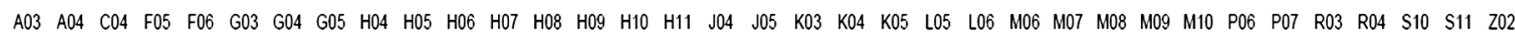

Students

FIG. 10. Ability summary chart. The chart gives the estimated ability parameters for each of the students listed on the horizontal axis. The horizontal broken lines mark the $95 \%$ confidence interval around the median of the ability scale. The black thick dashes mark person locations on the full PSCI reference scale. The thin dotted dashes represent the persons' locations on the subscales Application (Ap), Analysis (An), Evaluation (Ev), and PAIRS. A location at the top of the chart denotes good command of the concepts and skills addressed by the items in that subset, or high knowledge-coherence if the subset is PAIRS. A location at the bottom of the chart signals lack of appropriate knowledge to solve the items in that subset, or lack of knowledge-coherence if the subset is PAIRS. For the 35 students on this chart, there are numerous locations below the median of the Application-abilities. The instructor could infer that these students are not familiar with the concepts addressed by the set Application.

or he solved correctly (coherence category "deep"). One of the 27 students located at the median of the item scale had scattered knowledge and possible guessed some answers (coherence category "shallow"). One student located just below the median knew a part of the concepts well (coherence category "partial").

The finer discrimination provided by the subsets appears in the summary chart (Fig. 10). The summary chart allows the instructor who applies the PSCI-BETA-I to interpret the scores and make valid decisions for his class. In Spring 2013, there were numerous below median locations on the Application scale, signaling lack of knowledge pertaining to the concepts covered by this subset. Student M06, located below the median of the PSCI scale, had the highest location for the subset Evaluation; it could be inferred either that this student was familiar with some items in this subset or that guessing took place. Student M09, located at the median level, missed many items in the subset Application, which probably affected the coherence level indicated by the subset PAIRS. Student H08, above the median of the PSCI item scale and with "deep" knowledge-coherence, was missing knowledge contained in the subsets Application and Analysis. As student H08 had a top ability for inference processes (characteristic for the subset Evaluation), he could easily remediate the missing knowledge before the final exam.

The full results of the measurement with the PSCIBETA-I are presented in the Table IX. The rows in Table IX give the person parameter estimates for each student in testing. The first and last rows list the minimum and maximum possible values of these parameters; these extreme values were not observed but interpolated from the data in field testing. The first four columns in Table IX give the person parameter (location) estimated by the 18 -item testlet, the uncertainty of a person's location, and the knowledgecoherence category estimated with the 12-item subset PAIRS. The columns at the median of the Table IX give the five scores collected with the PSCI-BETA-I. The raw scores are useful for indicating how many questions were correctly answered in each subset. The scores for the subsets Application, Analysis, and Evaluation added together give the score for the 18-item subset. The right side of Table IX gives the person parameter estimates and their uncertainties for the subsets. Although the person parameter estimation on subsets with less than 10 items has about 1-logit size uncertainty, these subset-abilities are useful for discriminating among persons with the same PSCI-ability.

\section{Learning-gain estimation and baseline categories with the PSCI scale}

Nineteen students repeated the PSCI-ALPHA test after taking the PHYS3313 course in the traditional format. Table $\mathrm{X}$ provides the raw scores and the estimated abilities at pre- and postinstruction for these students. We calculated the ability-gains given by Eq. (6) and considered them significant only if the $95 \%$ confidence interval did not include the zero value. Significant gains were found for 


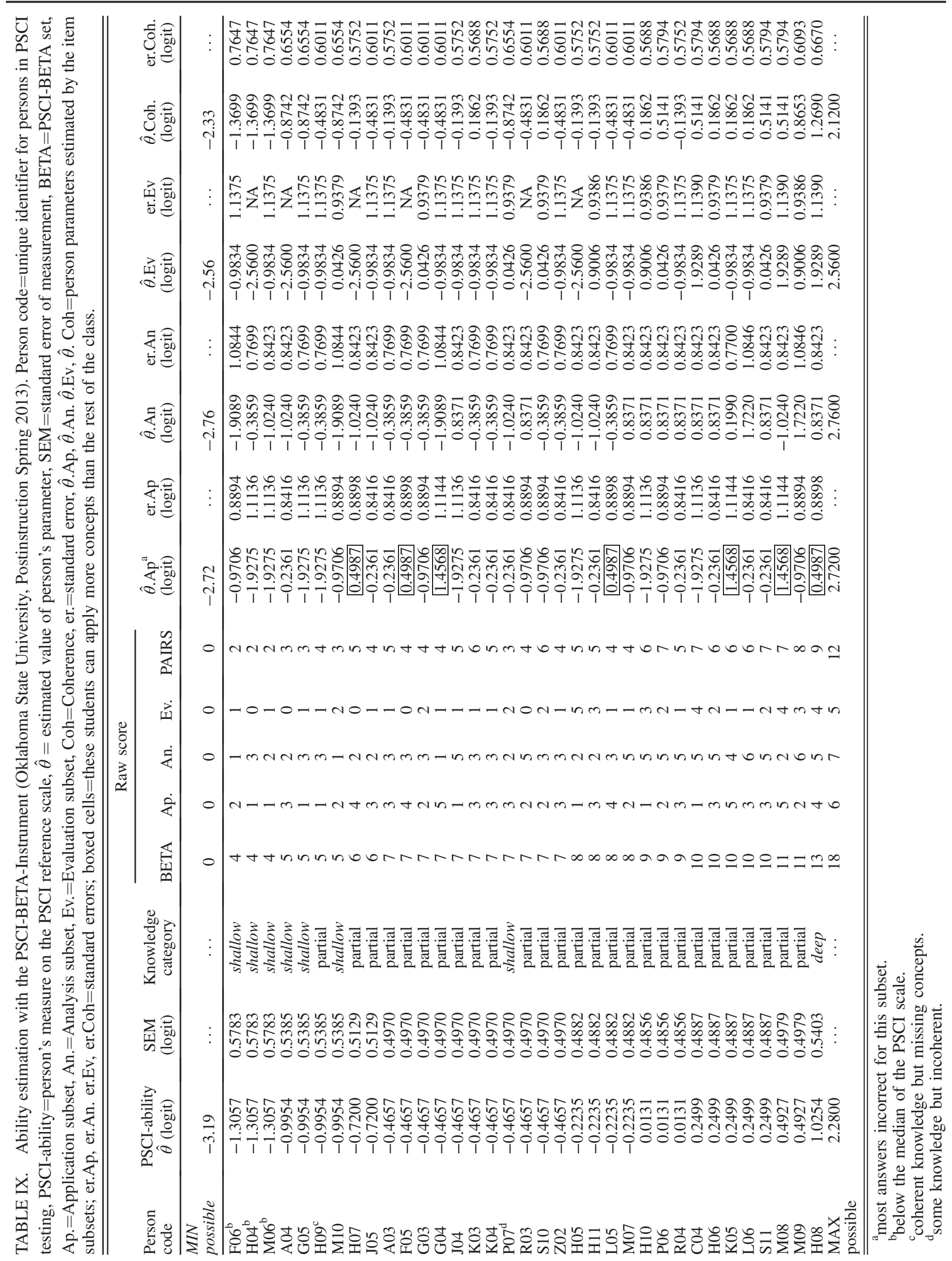


TABLE X. Baseline category and ability gain for the students repeating the PSCI testing at Oklahoma State University. Raw.PRE $(P O S T)=$ raw score for 30 -items at pre- and at postinstruction; $\hat{\theta}$.PRE (POST)=person's measure on the PSCI reference scale; Baseline=ability category before instruction; raw.Ap.PRE (POST) $=$ raw score for the 6-item application subset; $\hat{\theta}$.Ap.PRE $(\mathrm{POST})=$ person's measure on the application subscale; GAIN=ability gain, difference from PRE to POST; low(up) $95 \% \mathrm{CI}=$ lower and upper boundaries of the $95 \%$ confidence interval for the ability gain; boxed values=significant ability gain.

User raw.PRE $\hat{\theta}$.PRE Baseline Raw.Ap.PRE $\hat{\theta}$.Ap.PRE Raw.POST $\hat{\theta}$.POST Raw.Ap.POST $\hat{\theta}$.Ap.POST GAIN Low95\% CI Up95\% CI

\begin{tabular}{|c|c|c|c|c|c|c|c|c|c|c|c|c|}
\hline G01 & 6 & -1.4714 & Level 1 & 2 & -0.9706 & 10 & -0.7405 & 3 & -0.2361 & 0.7309 & 0.1167 & 1.3451 \\
\hline S07 & 6 & -1.4714 & Level 1 & 1 & -1.9275 & 12 & -0.4345 & 2 & -0.9706 & 1.0369 & 0.4319 & 1.6419 \\
\hline D01 & 7 & -1.2650 & Level 1 & 1 & -1.9275 & 8 & -1.0776 & 3 & -0.2361 & 0.1875 & -0.4255 & 0.8004 \\
\hline R02 & 7 & -1.2650 & Level 1 & 0 & -2.7216 & 10 & -0.7405 & 3 & -0.2361 & 0.5245 & -0.0714 & 1.1204 \\
\hline $\mathrm{S} 02$ & 7 & -1.2650 & Level 1 & 1 & -1.9275 & 7 & -1.2650 & 2 & -0.9706 & 0.0000 & -0.6259 & 0.6259 \\
\hline W04 & 8 & -1.0776 & Level 1 & 0 & -2.7216 & 6 & -1.4714 & 2 & -0.9706 & -0.3938 & -1.0246 & 0.2370 \\
\hline W06 & 8 & -1.0776 & Level 1 & 0 & -2.7216 & 9 & -0.9039 & 3 & -0.2361 & 0.1737 & -0.4162 & 0.7635 \\
\hline L02 & 8 & -1.0776 & Level 1 & 2 & -0.9706 & 9 & -0.9039 & 1 & -1.9275 & 0.1737 & -0.4162 & 0.7635 \\
\hline P05 & 8 & -1.0776 & Level 1 & 0 & -2.7216 & 15 & -0.0016 & 5 & 1.4568 & 1.0760 & 0.5082 & 1.6437 \\
\hline L01 & 9 & -0.9039 & Level 2 & 2 & -0.9706 & 10 & -0.7405 & 1 & -1.9275 & 0.1634 & -0.4087 & 0.7355 \\
\hline S09 & 9 & -0.9039 & Level 2 & 1 & -1.9275 & 15 & -0.0016 & 4 & 0.4987 & 0.9023 & 0.3450 & 1.4596 \\
\hline W05 & 10 & -0.7405 & Level 2 & 4 & 0.4987 & 8 & -1.0776 & 1 & -1.9275 & -0.3371 & -0.9194 & 0.2452 \\
\hline B05 & 10 & -0.7405 & Level 2 & 3 & -0.2361 & 12 & -0.4345 & 3 & -0.2361 & 0.3060 & -0.2483 & 0.8603 \\
\hline B01 & 10 & -0.7405 & Level 2 & 2 & -0.9706 & 12 & -0.4345 & 3 & -0.2361 & 0.3060 & -0.2483 & 0.8603 \\
\hline K01 & 12 & -0.4345 & Level 2 & 2 & -0.9706 & 16 & 0.1411 & 2 & -0.9706 & 0.5756 & 0.0360 & 1.1152 \\
\hline T02 & 12 & -0.4345 & Level 2 & 3 & -0.2361 & 16 & 0.1411 & 5 & 1.4568 & 0.5756 & 0.0360 & 1.1152 \\
\hline E02 & 12 & -0.4345 & Level 2 & 2 & -0.9706 & 17 & 0.2851 & 3 & -0.2361 & 0.7196 & 0.1782 & 1.2609 \\
\hline V02 & 14 & -0.1443 & Level 2 & 2 & -0.9706 & 16 & 0.1411 & 3 & -0.2361 & 0.2854 & -0.2496 & 0.6353 \\
\hline $\mathrm{J} 02$ & 17 & 0.2851 & Level 3 & 2 & -0.9706 & 18 & 0.4316 & 4 & 0.4987 & 0.1465 & -0.3951 & 0.6881 \\
\hline
\end{tabular}

seven of the repeated measures. Possible explanations for the low gains are that the level of the cognitive operations addressed by the PSCI-ALPHA was perhaps too difficult for our junior undergraduate engineering students, and that students were not motivated to perform in a post-instruction test administered two weeks before the final exam of the physics of semiconductors' class.

For better characterizing the student population, we employed the abilities estimated at preinstruction to assign the students to baseline knowledge categories. The persons located below -1 logit were assigned to the category Level 1 , those with $-1<\hat{\theta}_{\mathrm{PRE}}<0$ to Level 2 , those with $0<\hat{\theta}_{\mathrm{PRE}}<1$ to Level 3 and, finally, those with $\hat{\theta}_{\mathrm{PRE}}>1$ to Level 4; the higher the level number, the higher the ability. The ability for the subset application $\hat{\theta}_{\mathrm{Ap} \text {.PRE }}$ served as decision criterion for the persons within 0.3 logit below the cutoff. If, $\hat{\theta}_{\mathrm{Ap.PRE}}>0$ then the borderline person was assigned to the next baseline category. The distribution of the sample "PRE" in field testing obtained with this

TABLE XI. Baseline distribution and average learning gains of the sample in field testing. Baseline $=$ ability category before instruction; Level 1, Level 2, and Level $3=1$-logit long interval categories; $\langle\Delta \hat{\theta}\rangle=$ average ability gain on baseline categories, calculated by including only the matched measures; stdev = standard deviation of the average ability gain.

\begin{tabular}{lcccc}
\hline \hline Baseline & Count & Proportion & $\langle\Delta \hat{\theta}\rangle$ & Stdev \\
\hline Level 1 & 9 & $33 \%$ & 0.390 & 0.490 \\
Level 2 & 15 & $56 \%$ & 0.389 & 0.361 \\
Level 3 & 3 & $11 \%$ & 0.147 & $\ldots$ \\
total & 27 & $100 \%$ & & \\
\hline \hline
\end{tabular}

recipe is given in Table XI. Considering only the repeatedmeasures participants in field testing, we computed the average gain $\langle\Delta \hat{\theta}\rangle$ for each baseline category. The resulting values, listed in Table XI, were close to zero.

The baseline distribution can give the instructor that quick information needed about the class before starting the teaching activities. The PSCI test can thus act as a fast litmus test before instruction. Measuring learning gains with a 0.5 -logit uncertainty is still a useful measurement as long as the goal is to provide an objective diagnostic of the class of students before the last week of the semester, and not to precisely rank students or instructors.

The authors had to write and calibrate new items that would extend the range of measurement of the PSCI instrument. For targeting the typical population of the PHYS3313 classes at OSU, the supplementary items should be below the -0.6 logit average ability measured during field testing. Administering testlets centered on target would yield more precise ability measurements with the PSCI scale. The subsets Application, Analysis, and Evaluation will serve as anchors for linking the new items into the PSCI test bank.

\section{MML IRT modeling results}

The PSCI test was built to measure multiple lower order facets of the higher order dimension "composite-ability to operate with basic conceptual knowledge of semiconductors". We expected that the responses addressing the same concept category are correlated to each other [34]. The items in each concept category were analyzed separately using two-and three-parameter (2PL and 3PL) IRT models. The marginal maximum likelihood (MML) ltm $\mathrm{R}$ package 
$[67,68]$ was employed for this type of modeling. ${ }^{15}$ While the Rasch model (1PL IRT) considers all items equally discriminating, the 2PL and 3PL IRT models have the advantage of being able to account for the items' different discriminating powers [69]. The 2PL and 3PL modeling provided a "parameters fit the data" type of estimation, which was opposite to the Rasch's parametrization "data must fit the model". The 2PL IRT model [70] had a good fit on the entire PSCI-ALPHA and also on separate groups of four or more items belonging to the same concept category. We interpreted the fit on each concept category as a confirmation that the items in that group address the same concept.

We compared the results of the separable-parameters CML approach to the outcomes offered by a panoply of MML models in the package $\mathrm{ltm}$. The 1PL IRT model from the $l t m$-MML package and the $e R m$-CML method ranked the 18 items of the PSCI-BETA identically. Compared to the CML, the MML method inflated the distance between two adjacent items by a factor of 4.6. The average standard error was 8.1 times greater for MML than for CML. We concluded that the CML method functioned better for our small calibration sample. In addition, the MML method may have limited generalizability to other samples of persons because it provides a person dependent parametrization of the items.

\section{Validity of the PSCI scale and subscales}

Validity certifies whether or not the test measures what it says it measures, as well as what interpretations can be inferred from the scores on the test [71-73]. Evidence that supports the interpretation of the measurements via the PSCI scale was accumulated during all the stages of the test development process described through this paper. The relevancy and clarity of the initial pool of items was investigated by expert raters, who provided evidence of content-related validity. The interviews helped validate that the novices' interpretations were consistent with the intended meaning of each item. Evidence regarding the knowledge and cognitive processes employed for item solving was collected after the pilot study by analyzing the responses to the test and interviews. Classical test analysis and IRT modeling were employed for qualitative confirmation of the test construct and internal structure validity. The good fit on the Rasch linear scale and the confirmation of the calibration invariance on an independent sample of persons in April 2013 provided evidence for reliability. The limited precision of the person measures was counterbalanced by a triangulation method.

\section{SUMMARY AND CONCLUSIONS}

An assessment instrument for a specialty course was built with the collective contribution of instructors from seven

\footnotetext{
${ }^{15}$ Exemplary outputs of the modeling with the package $\mathrm{ltm}$ are provided in the Supplemental Material [15].
}

universities. Although item calibration was performed on only 64 persons in field testing, we obtained an invariant scale of 30 items that can locate a person with a 0.4-logit uncertainty. Subsets of this 30-item set are useful for discriminating among persons with the same location. Any team of instructors teaching a small size specialty course could follow the recipe described in this paper to calibrate a bank of items on a sample smaller than 100 students.

We adopted the conditional likelihood maximization method as the best suited method for generating an invariant reference knowledge scale on a small size sample of persons, as opposed to the classical test theory and MML methods. The crucial benefit of the Rasch invariantly calibrated scale is that persons are not compared among themselves but with fixed located items. Our PSCI instrument has the rigidity of a yardstick. The onedimensional PSCI-scale measures the unique construct "composite-ability to operate with basic conceptual knowledge of physics of semiconductors". An important component of the PSCI-construct is the procedural knowledge, i.e., "knowledge of inferring information from graphs and plots".

One of our goals was to characterize the student population enrolled each semester for each instructor. Our solution was to employ the person parameters estimated at pre-instruction for assigning the students to baseline-knowledge categories. The baseline distribution gives the instructors that information about the class that they need for planning future teaching activities: the PSCI test acts as a fast litmus test before instruction.

Because the items were calibrated on a small sample, the person parameter estimations implied sizable uncertainties, i.e., 0.5-logit at the center of the PSCI-BETA 18-item scale. For obtaining a sharper discrimination between person locations, we introduced subscales that track knowledge directly at three different cognitive levels. We devised a method for estimating knowledge-coherence and signaling possible guessing. Information on gaps in students' knowledge or their lack of knowledge-coherence represents crucial information for an instructor who is revisiting selected concepts in class before an exam.

The person-free calibrated PSCI scale leads to an objective measure of the learning gain at the end of the instructional period for each individual. The objectivity of this learning gain arises from comparing successive locations of the persons on a fixed reference item scale. Instead of the $\langle g\rangle$ metric, we proposed reporting an average abilitygain $\langle\Delta \hat{\theta}\rangle$ separately for each category of persons situated at preinstruction at the same location on the item scale (separately for each baseline category). The average abilitygain metric is precise enough to compare instructors' effectiveness across the baseline categories.

The Rasch invariantly calibrated PSCI test can be employed on groups of persons with a lower or higher ability than the sample in field testing. Another benefit of 
the Rasch calibration is scalability: the PSCI test bank may be expanded with new items of targeted content and difficulty that would extend the range of measurement $[36,37]$. To target the typical population of the PHYS3313 classes at OSU, the supplementary items that we are adding are below the -0.6 logit average ability measured during field testing. The subsets Application, Analysis, and Evaluation serve as anchors for linking the new items into the PSCI testbank.

The PSCI-BETA-I described in this paper can be applied to other groups of persons who are enrolled or have been enrolled in an introductory semiconductor devices course. The PSCI-BETA-I can be administered as a formative diagnostic tool or as a placement test before an advanced course of semiconductor devices. The subsets in the PSCIBETA-I facilitate the rapid identification of the missing concepts or cognitive abilities. The partial score for the item-subset PAIRS accounts for the quality of knowledge. A coded summary chart facilitates a quick judgment to be made about the general performance of the class of students and about the knowledge consistency of the individuals. Any instructors interested in administering the PSCI-BETA-I to their students may contact us to receive a secure copy of the test and the score-to-chart conversion codes.

\section{ACKNOWLEDGMENTS}

The authors are especially grateful to Alan Cheville, Seth Bank, Robert Hauenstein, and Jerzy Krasinski for their contribution to the writing of the initial pool of items, and to Jeremy Penn from the Office of Assessment at the North Dakota State University for the discussions on the statistical modeling. Special thanks to Mario Borunda, Alexander Khanov, and Flera Rizatdinova from Oklahoma State University for applying versions of the PSCI test to their students. Hearty thanks go to Andreea Botezatu from the Texas A\&M University for English proofing and to Suzanne Lidström from Uppsala University for her comments on the manuscript.
[1] A. Cheville, Engineering Students for the 21st Century: Student Development Through the Curriculum (Oklahoma State University, Stillwater, OK, 2010).

[2] A. Cheville and C. Bunting, Engineering Students for the 21st Century: Student Development through the Curriculum, Adv. Engineering Educ. 2, 1 (2011).

[3] AAPT, PhysPort. 2017; available from https://www .physport.org/assessments/.

[4] D. Hestenes, M. Wells, and G. Swackhamer, Force Concept Inventory, Phys. Teach. 30, 141 (1992).

[5] R. Hake, The Impact of Concept Inventories On Physics Education and It's Relevance For Engineering Education, Proceedings of the National Meeting on STEM Concept Inventories (National Science Foundation, Washington, DC, 2011).

[6] J. Nicolora, D. Rosado, and R. Gasper, Concept Inventories in Engineering Science and Design (Worchester Polytechnic Institute, Worcester, Massachusetts, 2012).

[7] J. Libarkin, Concept Inventories in Higher Education Science, National Science Council Promising Practices in Undergraduate STEM Education (The National Academy of Sciences, Washington, DC, 2008).

[8] F. Reif and S. Allen, Cognition for Interpreting Scientific Concepts: A Study of Acceleration, Cognit. Instr. 9, 1 (1992).

[9] F. Reif, Applying Cognitive Science to Education: Thinking and Learning in Scientific and Other Complex Domains (Bradford Books, The MIT Press, Cambridge, Massachusetts, 2008), ISBN 9780262182638.

[10] T. Husén and T. N. Postlethwaite, The International Encyclopedia of Education, 2nd ed. (Pergamon, Elsevier Science, New York, 1994).
[11] L. W. Anderson and D. R. Krathwohl, A Taxonomy for Learning, Teaching, and Assessing: A Revision of Bloom's Taxonomy of Educational Objectives (Longman, New York, 2001).

[12] D. R. Krathwohl, A Revision of Bloom's Taxonomy: An Overview, Theory Into Practice 41, 212 (2002).

[13] G. Rasch, Studies in Mathematical Psychology: I. Probabilistic Models for Some Intelligence and Attainment Tests (Oxford, Nielsen \& Lydiche, England 1960), p. 184.

[14] F. M. Lord, Applications of Item Response Theory to Practical Testing Problems (Erlbaum Associates, Hillsdale, NJ, 1980).

[15] See Supplemental Material at http://link.aps.org/ supplemental/10.1103/PhysRevPhysEducRes.14.010102 for additional information for the PSCI study.

[16] G. Rasch, Probabilistic Models for Some Intelligence and Attainment Tests (University of Chicago Press, Denmark, 1980), pp. 3-12, ISBN 9780226705538.

[17] S. W. Anderson, J. Libarkin et al. GEOSCIENCE CONCEPT INVENTORY Wiki. 2013 [cited 2013 6-14], http:// geoscienceconceptinventory.wikispaces.com/.

[18] J. C. Libarkin et al., Revisiting the Geoscience Concept Inventory: A call to the community, GSA Today 21, 26 (2011).

[19] C. S. Wallace and J. M. Bailey, Do concept inventories actually measure anything, Astron. Educ. Rev. 9, 010116 (2010).

[20] B. D. Wright and M. H. Stone, Best Test Design (MESA Press Chicago, 1979).

[21] J. M. Linacre, Sample size and item calibration stability, Rasch Measurement Trans. 7, 328 (1994); www.rasch.org/ $\mathrm{rmt} / \mathrm{rmt} 74 \mathrm{~m} . \mathrm{htm}$. 
[22] J. S. Aslanides and C. M. Savage, The Relativity Concept Inventory: Development, analysis and results, Phys. Rev. ST Phys. Educ. Res. 9, 010118 (2013).

[23] B. Wright and M. Mok, Understanding Rasch measurement: Rasch models overview, J. Appl. Measure. 1, 83 (2000).

[24] P. Mair and R. Hatzinger, Extended Rasch Modeling: The $e R m$ Package for the Application of IRT Models, R. J. Stat. Softw. 20, 1 (2007).

[25] G. Fischer, Derivations of the Rasch Model, in Rasch Models, edited by G. Fischer and I. Molenaar (Springer, New York, 1995), p. 16.

[26] E. B. Andersen, Sufficient statistics and latent trait models, Psychometrika 42, 69 (1977).

[27] G. Fischer, Derivation of the Rasch Model, in Rasch Models, edited by G. Fischer and I. Molenaar (Springer, New York, 1995), p. 15-26.

[28] E. B. Andersen, What Georg Rasch Would Have Thought about this Book, in Rasch Models, edited by G. Fischer and I. Molenaar (Springer, New York, 1995), p. 383-390.

[29] P. W. Holland, On the sampling theory foundations of the item response theory models, Psychometrika 55, 577 (1990).

[30] G. H. Fischer, On the existence and uniqueness of maximum-likelihood estimates in the Rasch model, Psychometrika 46, 59 (1981).

[31] C. A. W. Glas and N. D. Verhelst, Testing the Rasch Model in Rasch Models, edited by G. Fischer and I. Molenaar (Springer, New York, 1995), p. 69.

[32] I. W. Molenaar, Estimation of Item Parameters, in Rasch Models, edited by G. Fischer and I. Molenaar (Springer, New York, 1995), p. 44-47.

[33] P. Mair, R. Hatzinger, and M. J. Maier, The R Package $e R m, 2017$, CRAN R project, https://cran.r-project.org/ web/packages/eRm.

[34] B. D. Wright and G. N. Masters, Rating Scale Analysis: Rasch Measurement (MESA Press, Chicago, 1982).

[35] E. B. Andersen, Asymtotic Properties of Conditional Maximum Likelihood Estimators, J. R. Stat. Soc. 32, 283 (1970).

[36] H. Hoijtink and A. Boomsma, On Person Parameter Estimation in the Dichotomous Rasch Model, in Rasch Models, edited by G. Fischer and I. Molenaar (Springer, New York, 1995), p. 53-68.

[37] J. Njiru and J. Romanoski, Development and calibration of physics items to create an item bank, using the Rasch measurement model, Int. J. Teach. Learn. Higher Educ. 14, 3 (2007).

[38] Innovations in Computerized Assessment, edited by $\mathrm{F}$. Drasgow and J. B. Olson-Buchanan (LEA, Mahwah, NJ, 1999).

[39] G. H. Fischer, Gain Scores Revisited Under an IRT Perspective, in Essays on Item Response Theory, edited by A. Boomsma, M. A. J. v. Duijn, and T. A. B. Snijders (Springer, New York, 2001), p. 43-68.

[40] G. H. Fischer, The precision of gain scores under an item response theory perspective: A comparison of asymptotic and exact conditional inference about change, Appl. Psychol. Meas. 27, 3 (2003).

[41] J. Rost, A note on the correlation of gain scores and achievement level, Polskie Forum Psychologiczne 18, 5 (2013).
[42] R. Hake, Interactive-engagement versus traditional methods: A six-thousand-student survey of mechanics test data for introductory physics courses, Am. J. Phys. 66, 64 (1998).

[43] V. Colletta and J. Phillips, Interpreting FCI scores: Normalized gain, preinstruction scores, and scientific reasoning ability, Am. J. Phys. 73, 1172 (2005).

[44] L. Bao, Theoretical comparisons of average normalized gain calculations, Am. J. Phys. 74, 917 (2006).

[45] B. L. Anderson and R. L. Anderson, Fundamentals of Semiconductor Devices (McGraw-Hill Higher Education, Boston, 2005).

[46] Electronic Devices and Circuit Theory, 9th ed., edited by R. L. Boylestad and L. Nashelsky (Prentice Hall, New York, 2006), p. 21.

[47] J. E. Carroll, Rate equations in Semiconductor Electronics (Cambridge University Press, Cambridge, England, 1985).

[48] D. A. Neamen, Semiconductor Physics and Devices, 3rd ed. (McGraw-Hill, New York, 2003).

[49] R. F. Pierret, Semiconductors Fundamentals, 2nd ed. Modular Series on Solid State Devices, edited by R.F. Pierret and G. W. Neudeck (Addison-Wesley, Reading MA, 1989), Vol. I, p. 3.

[50] B. Streetman and S. Banerjee, Solid State Electronic Devices, 5th ed. (Prentice Hall Upper Saddle River, NJ, 2000).

[51] S. M. Sze, Physics of Semiconductor Devices, 2nd ed. (John Wiley \& Sons, New York, 1981).

[52] S. M. Sze, Semiconductor Devices: Pioneering Papers, edited by A. T. B. Laboratories (World Scientific, Singapore, 1991).

[53] B. V. Zeghbroeck, Principles of Semiconductor Devices (2007), online book, http://ece-www.colorado.edu/ bart/ book/.

[54] W. Shokley, The quantum physics of solids, Bell Syst. Tech. J. 17, 1184 (1939).

[55] PhET_Interactive_Simulations, Photoelectric Effect, https://phet.colorado.edu University of Colorado, Boulder.

[56] L. Ding, Applying Rasch theory to evaluate the construct validity of brief electricity and magnetism assessment, AIP Conf. Proc. 1413, 175 (2012).

[57] L. Ding, Seeking missing pieces in science concept assessments, Phys. Rev. ST Phys. Educ. Res. 10, 010105 (2014).

[58] L. Guttman, A basis for analyzing test-retest reliability, Psychometrica 10, 255 (1945).

[59] R, Foundation for Statistical Computing 2017; Available from: https://cran.r-project.org/.

[60] E. B. Andersen, The numerical solution of a set of conditional estimation equations, J. R. Stat. Soc. Ser. B 34, 42 (1972).

[61] R. Smith, Reliability and validity, Introduction to Rasch Measurement (JAM Press, Maple Grove, MN, 2004), p. 93-122.

[62] R. Freund and W. Wilson, Statistical Methods, 2nd ed. (Academic Press, New York, 2003).

[63] The Distribution of Chi-Square, edited by E. B. Wilson and M. M. Hilferty (National Academy of Sciences, Washington, DC, 1931).

[64] X. Liu, Using and developing measurement instruments in science education, Science and Engineering Education 
Sources, edited by C. U. Calvin and S. Kalman (Information Age Publishing, , Charlotte, NC, 2010).

[65] E. Timminga and J. J. Adema, Test Construction Item Banks, in Rasch Models, edited by G. Fischer and I. Molenaar (Springer, New York, 1995), p. 111-127.

[66] G. H. Fisher and I. Ponocny, Extended Rating Scale and Partial Credit Models for Assessing Change, in Rasch Models, edited by G. Fischer and I. Molenaar (Springer, New York, 1995), p. 353-370.

[67] D. Rizapoulos, CRAN_Package ltm, 2012, CRAN.RProject, https://cran.r-project.org/web/packages/ltm/ltm.pdf.

[68] D. Rizopulos, Latent Trait Models under IRT, 2012, CRAN, https://cran.r-project.org/web/packages/ltm/index.html.

[69] T. Rusch, P. Mair, and R. Hatzinger, Psychometrics with R: A Review of CRAN Packages for Item Response
Theory, in Discussion Paper Series, edited by R. Dittrich et al. (Center for Empirical Research Methods, Vienna University of Economics and Business, Vienna, 2013).

[70] D. Rizopoulos, Itm: An R package for latent variable modeling and item response theory analyses, J. Stat. Softw. 17 (2006).

[71] L. Crocker and J. Algina, Introduction to Classical and Modern Test Theory (Holt, Rinehart, and Winston, New York, 1986), p. 217-236.

[72] E. W. Wolfe and E. V. J. Smith, Instrument development tools and activities for measure validatiom using Rasch models: Part II-Validation activities, J. Appl. Meas. 8, 204 (2007).

[73] AERA, APA, and NCME, Standards for Educational and Psychological Testing 2014, ISBN: 978-0-935302-35-6. 